Hydroécol. Appl. (1997) Tome 9 Vol. 1-2, pp. 85-114

\title{
Evolution temporelle des émissions gazeuses et des profils de gaz dissous Estimation du bilan de carbone de la retenue de Petit-Saut deux ans après sa mise en eau
}

\author{
Evolution over time of gaseous emissions and dissolved \\ gas profiles. Estimation of the carbon budget in Petit-Saut \\ resenoir, two years after filling
}

\author{
C. Galy-Lacaux (1), R. Delmas (1), J.-F. Dumestre(2) \\ et Sandrine Richard(3) \\ (1) Laboratoire d'Aérologie (UMR CNRS/UPS 5560), O.M.P, 14, avenue Edouard-Belin, \\ 31400 Toulouse, France. \\ (2) CESAC (Centre d'Ecologie des Systèmes Aquatiques Continentaux) \\ UMR/UPS C 5576 Université Paul-Sabatier, 118, route de Narbonne, \\ 31062 Toulouse cedex, France.
}

(3) Laboratoire Environnement, Aménagement de Petit-Saut, Société HYDRECO, BP 823, 97388 Kourou cedex, Guyane.

\begin{abstract}
Résumé. - Les émissions de méthane, de gaz carbonique et d'hydrogène sulfuré de la retenue hydroélectrique de Petit-Saut sur la rivière Sinnamary, en Guyane, ont été mesurées pendant une période de 2 ans. Depuis le début du remplissage (Janvier 1994), $300 \mathrm{~km}^{2}$ de forêt ont été submergés. Les èmissions par diffusion et par effervescence à l'interface eau-atmosphère, par dégazage de l'eau rejetée en aval du barrage, ainsi que les stocks de gaz en solution, et leurs évolutions, ont été étudiés. Des émissions maximales de 800 tonnes de $\mathrm{CH}_{4}$ par jour furent atteintes en février 1995; elles étaient associées à des concentrations moyennes de $\mathrm{CH}_{4}$ dissous dans la colonne d'eau de $14 \mathrm{mg} \cdot \mathrm{l}^{-1}$. L'oxydation biologique du méthane dans l'eau conduit à une consommation d'oxygène dans le lac et dans la rivière en aval du barrage. Les émissions totales de $\mathrm{CH}_{4}$ et $\mathrm{CO}_{2}$ depuis janvier 1994, sont calculées à partir de l'ensemble des données expérimentales qui permettent également d'évaluer la perte de carbone du réservoir depuis la mise en eau.
\end{abstract}

Mots clés. - barrage hydroélectrique, méthane, gaz carbonique, hydrogène sulfuré, oxygène. 
Abstract. - Methane, carbon dioxide and hydrogen sulfide emissions from the reservoir above Petit-Saut hydroelectric dam on the Sinnamary River in French Guiana, were measured over a 2-year period. Since the beginning of filling of the reservoir (January 1994), $300 \mathrm{~km}^{\text {? }}$ of tropical forest have been submerged. Emissions of $\mathrm{CH}_{4}$ by diffusion and bubbling into the atmosphere or by degasing of the water released into the river as well as the stock of dissolved gases in the lake, and their evolution over time, have been studied. Maximum emissions of 800 Tons $\left(\mathrm{CH}_{4}\right)$ per day were attained in February 1994, corresponding to mean dissolved $\mathrm{CH}_{4}$ concentrations of $14 \mathrm{mg}^{\mathrm{l}^{-1}}$ in the water column. The biological oxidation of methane results in high oxygen consumption in the water of the lake and of the river. Total emissions of $\mathrm{CH}_{4}$ and $\mathrm{CO}_{2}$ from January 1994, are calculated on the basis of the complete data set which also enables calculation of the total carbon loss since filling of the reservoir.

Key words. - hydroelectric dams, methane, carbon dioxide, hydrogen sulfide, oxygen.

\section{INTRODUCTION}

Depuis deux siècles environ, les activités humaines contribuent à une modification sensible de la composition de l'atmosphère et notamment des concentrations des gaz à effet de serre. Un effort important d'évaluation des sources anthropogéniques est réalisé, pays par pays, dans le cadre de l'inventaire IPCC (International Panel for Climate Change). La modification de l'utilisation des sols est une des causes importantes de perturbations de la composition chimique de l'atmosphère. Cette modification ne se résume pas à la déforestation pour le développement de l'agriculture, elle peut avoir des causes très diverses. Le remplacement d'une forêt par un plan d'eau crée par l'homme, comme dans le cas des barrages hydroélectriques en zone équatoriale, modifie profondément le milieu et perturbe les échanges biosphère atmosphère en influant à la fois sur les sources et les puits de ces composés, et notamment des gaz à effet de serre (GES) tels que le gaz carbonique et le méthane. Les augmentations de ces deux gaz sont une des principales causes de l'effet de serre additionnel (Shine et al., 1990), mais le méthane est aussi un des précurseurs de l'ozone troposphérique. Par ailleurs, en raison de sa longue durée de vie, il intervient dans la chimie de la stratosphère en contrôlant partiellement le bilan de la vapeur d'eau et en limitant le pouvoir catalytique des atomes de chlore (Khalil, 1995).

Dans les lacs de barrage, la décomposition de la biomasse submergée conduit à la libération, sous forme de gaz carbonique, du carbone stocké dans la biomasse; toutefois les conditions d'anaérobiose régnant au fond de la retenue favorisent la production de méthane et d'hydrogène sulfuré et leur éventuelle émission dans l'atmosphère (Conrad, 1989). Des données sur les lacs naturels (Brinkman et Santos, 1974) ou les forêts inondées 
(Devol et al., 1988; Bartlett et al. 1988, Smith and Lewis, 1992, Tathy et al., 1992; Wassmann et al., 1992) sont disponibles; cependant il existe très peu de données concernant les lacs de barrage. Quelques études ont été menées sur les aménagements hydroélectriques dans les pays voisins de la Guyane (Brésil, Surinam) (Heide, 1982; Electronorte, 1993; Pereira, 1994; Fearnside, 1995) ou bien en Afrique (Balon et Coche, 1974) mais aucune étude expérimentale sur la quantification des différentes émissions gazeuses par une retenue d'eau artificielle en milieu tropical n'a été réalisée. Ce type de travail est effectué sur les grands réservoirs hydroélectriques du Canada (Chamberland, 1992; Rudd et al., 1993; Kelly et al 1994; Duchemin et al., 1995). Les lacs de barrages construits en zone de forêt tropicale sont susceptibles de constituer des sources potentielles importantes de gaz à effet de serre en raison des conditions climatiques et des quantités de biomasse submergées, toutefois aucune donnée expérimentale n'est actuellement disponible pour étayer cette affirmation. Cependant les émissions effectives ne peuvent être évaluées à partir de considérations simples uniquement basées sur des bilans de carbone. En effet, d'une part la biodégradabilité en condition d'anoxie des différents compartiments de la matière organique d'une forêt est très variable, allant de quelques jours pour la biomasse microbienne à plusieurs siècles pour la fraction ligneuse; d'autre part les gaz réduits tels que $\mathrm{CH}_{4}$ et $\mathrm{H}_{2} \mathrm{~S}$ pro- duits au niveau du sédiment peuvent subir une oxydation chimique ou biologique dans la colonne d'eau qui péut réduire ou supprimer leur émission dans l'atmosphère (Rudd et Taylor, 1980, Cicerone et Oremland, 1988, Conrad, 1989; Oremland and Culbertson, 1992).

Le barrage de Petit-Saut en Guyane, construit sur la rivière Sinnamary, a été mis en eau en janvier 1994. II a atteint sa côte maximale ( $35 \mathrm{~m}$ ) en juillet 95 submergeant environ $300 \mathrm{~km}^{2}$ de forêt. L'évolution de la production de méthane, de gaz carbonique et d'hydrogène sulfuré dans le lac a été suivie depuis le début de la mise en eau (Galy-Lacaux et al., 1996; Galy-Lacaux, 1996, GalyLacaux et al., 1997a). Cette production se traduit par une émission à la surface du lac, par une émission consécutive au dégazage de l'eau rejetée dans la rivière, et par une augmentation progressive des teneurs en gaz dissous dans la masse d'eau. La production de méthane contribue de façon importante à la consommation d'oxygène dans l'eau du lac et de la rivière en aval du barrage. Pour étudier ces phénomènes, différentes techniques de mesure des flux gazeux ont été mises en œuvre. L'interprétation des mesures de flux net $\left(\mathrm{CH}_{4}, \mathrm{CO}_{2}\right)$ à la surface du lac a nécessité une étude des phénomènes de réoxydation dans la colonne d'eau des composés réduits formés au niveau des sédiments en collaboration avec le Centre d'Etudes des Ecosystèmes Aquatiques de Toulouse (C.E.S.A.C); et s'appuie sur la me- 
sure des caractéristiques physico-chimiques de l'eau le long de profils entre la surface et le fond du lac pour différentes profondeurs d'eau.

Dans cet article, nous présentons essentiellement les résultats concernant le suivi des émissions de $\mathrm{CH}_{4}$, $\mathrm{CO}_{2}$ et dans une moindre mesure $\mathrm{H}_{2} \mathrm{~S}$ et $\mathrm{N}_{2} \mathrm{O}$ et du stock de gaz dissous, au cours des 2 premières années après la mise en eau du barrage. L'intégration de l'ensemble des données recueillies permet de dresser le bilan du carbone au niveau de la retenue et donc d'estimer la fraction de matière organique dégradée pendant la période considérée.

\section{SITES ET MÉTHODES}

\subsection{Le barrage de Petit-Saut}

Le barrage de Petit-Saut est implanté sur la rivière Sinnamary. II a été construit sous la responsabilité du Centre National d'équipement $\mathrm{Hy}$ draulique (CNEH) d'Electricité de France (EDF), et mis en eau en janvier 1994. La retenue a atteint sa côte maximale de $35 \mathrm{~m}$ en juillet 1995 . Le volume d'eau stocké à la côte maximale est de 3,5 milliard de $\mathrm{m}^{3}$. La puissance hydroélectrique installée est de $114 \mathrm{MW}$, délivrée par 4 groupes. Le débit turbiné à la puissance maximale est de $436 \mathrm{~m}^{3} . \mathrm{s}^{-1}$ et le débit moyen du Sinnamary est de $260 \mathrm{~m}^{3} \cdot \mathrm{s}^{-1}$. Au cours du remplissage du réservoir, qui a duré 18 mois, un débit minimum de $100 \mathrm{~m}^{3} \cdot \mathrm{s}^{-1}$ a toujours été maintenu en aval de la digue. Après que le lac ait atteint sa côte maximale, le rapport entre le débit moyen du fleuve et le volume du réservoir donne un temps de renouvellement moyen de l'eau dans la retenue d'environ 6 mois (Sissakian, 1992).

Six campagnes de recherche ont été effectuées sur le lac de Petit-Saut depuis la mise en eau de la retenue: en mars, septembre et décembre 1994, février, mai et septembre 1995. Au cours de ces campagnes ont été mesurés: les flux d'émission de $\mathrm{CH}_{4}$, de $\mathrm{CO}_{2}$, d' $\mathrm{H}_{2} \mathrm{~S}$ et de $\mathrm{N}_{2} \mathrm{O}$ à l'interface eau-atmosphère, les concentrations en gaz dissous $\left(\mathrm{CH}_{4}, \mathrm{CO}_{2}, \mathrm{H}_{2} \mathrm{~S}, \mathrm{~N}_{2} \mathrm{O}\right.$ et $\mathrm{O}_{2}$ ) et les caractéristiques physicochimiques (Conductivité, pH, potentiel d'oxydoréduction, température) de la colonne d'eau. Trois sites localisés en amont sur le lac à $40 \mathrm{~km}$ (CriqueBonne-Nouvelle-CBN), $20 \mathrm{~km}$ (Forêt inondée - FI) et $300 \mathrm{~m}$ (Bouée PetitSaut - BPS) de la digue ont été explorés systématiquement à chaque campagne. Depuis mars 1995, un suivi hebdomadaire des concentrations de $\mathrm{CH}_{4}$ et $\mathrm{O}_{2}$ dans la colonne d'eau est réalisé par le Laboratoire Environnement de Petit-Saut sur le troisieme site (BPS). En aval, des transects sur la rivière Sinnamary, à partir de la digue du barrage vers Pointe Combi (situé à $40 \mathrm{~km}$ du barrage et proche de l'estuaire) ont été réalisés lors d'expériences spécifiques en vue d'étudier les différentes composantes des flux de méthane et la relation méthane oxygène de façon détaillée. 


\subsection{Méthodes expérimentales}

\section{- Flux diffusif à la surface du lac}

Les mesures de flux à la surface du lac sont effectuées par la technique des chambres statiques. Une enceinte en inox, rectangulaire $(50 \times 50 \mathrm{~cm})$, d'un volume de $50 \mathrm{l}$, munie d'un flotteur, est déposée à la surface de l'eau. Un circuit d'air fermé, entretenu par une pompe péristaltique permet d'homogénéiser l'air dans l'enceinte avant de réaliser des prélèvements. Pour la mesure des flux de $\mathrm{CH}_{4}$ et $\mathrm{CO}_{2}$, les prélèvements d'air dans l'enceinte sont réalisés au moyen d'une seringue, toutes les 15 minutes. L'air prélevé $(50 \mathrm{ml})$ est injecté dans des tubes de chromatographie en verre préalablement vidés, munis de septum en téflon. Pour assurer une étanchéité parfaite le septum des tubes est recouvert d'une couche de vernis après le prélèvement. Les tubes sont stokés à l'abri de la lumière. Des tests réalisés en laboratoire pour des expériences antérieures ont montré que la concentration de méthane dans les tubes ainsi conditionnés n'évolue pas pendant plusieurs mois. La variation de concentration en $\mathrm{CH}_{4}$, et $\mathrm{CO}_{2}$ qui permet de déduire les flux d'émission à partir de la pente de la droite obtenue est déterminée par chromatographie en phase gazeuse (Delmas et al., 1992).

\section{- Flux ébullitif à la surface du lac}

Le piégeage des bulles est réalisé à l'aide d'entonnoirs renversés et partiellement submergés à la surface de l'eau (Keller et al., 1994). Ces enton- noirs remplis d'eau flottent grâce à une plaque de polystyrène située au niveau du col. L'entonnoir en polyéthylène est large de $28 \mathrm{~cm}$ à la base et de 2,1 cm au niveau du col. Le col est bouché par un septum qui permet à l'aide d'une seringue graduée de prélever le volume de gaz recueilli dans l'entonnoir. Les bulles qui remontent dans la colonne d'eau entrent par la base de l'entonnoir et déplacent un volume d'eau équivalent dans le $\mathrm{col}$, où vont s'accumuler les gaz. Ici, l'objectif est de supprimer l'interface eau-atmosphère de manière à ne recueillir que l'émission due au phénomène d'effervescence. L'échantillonnage est réalisé au bout de deux heures environ. Le volume de gaz prélevé à l'aide d'une seringue est injecté dans des tubes de verre préalablement vidés. L'investigation de chaque site de mesures est réalisée à l'aide de 5 entonnoirs attachés à un guide et régulièrement espacés entre deux points afin d'augmenter la valeur statistique des résultats obtenus.

\section{- Profils de concentration dans l'eau}

Pour déterminer les profils de gaz $\left(\mathrm{CH}_{4}, \mathrm{CO}_{2}\right.$ et $\left.\mathrm{N}_{2} \mathrm{O}\right)$ en solution, l'eau est aspirée à différentes profondeurs à l'aide d'une pompe péristaltique. $20 \mathrm{ml}$ d'eau sont prélevés dans le tube d'aspiration au moyen d'une seringue et injectés dans les tubes de chromatographie de $50 \mathrm{ml}$ sous vide. L'eau est donc dégazée; avant l'analyse les tubes sont rééquilibrés à la pression atmosphérique. La concentration en $\mathrm{CH}_{4}, \mathrm{CO}_{2}$ et $\mathrm{N}_{2} \mathrm{O}$ dans l'atmosphère surnageante ("heads- 
pace"), en équilibre avec l'eau, est dosée par chromatographie en phase gazeuse. Le coefficient de partage (Loi de Henry) est utilisé pour déterminer la quantité de gaz résiduel dans l'eau et donc calculer la quantité totale de gaz dissous de l'échantillon (Sigg et al., 1992).

\section{- Analyses chromatographiques de $\mathrm{CH}_{4}, \mathrm{CO}_{2}$ et $\mathrm{N}_{2} \mathrm{O}$}

Les analyses de $\mathrm{CH}_{4}$ et de $\mathrm{CO}_{2}$ sont réalisées à l'aide d'un chromatographe Hewlett Packard HP 5890A équipé d'une colonne semi capillaire Porapak $Q$ et d'un détecteur à ionisation de flamme pour la mesure du $\mathrm{CH}_{4}$, et d'une colonne remplie Poraplot $Q$ et d'un détecteur à conductivité thermique pour la mesure du $\mathrm{CO}_{2}$. L'étalonnage est réalisé au moyen d'un étalon certifié d'Air Liquide à $3000 \mathrm{ppm}$ de $\mathrm{CH}_{4}$ et $30000 \mathrm{ppm}$ de $\mathrm{CO}_{2}$. Un étalon plus faiblement concentré à $2 \mathrm{ppm}$ de $\mathrm{CH}_{4}$ et à $400 \mathrm{ppm}$ de $\mathrm{CO}_{2}$ est utilisé pour les mesures de flux gazeux. Les analyses sont effectuées au Laboratoire Environnement de Petit-Saut dans les trois jours qui suivent le prélèvement. Les analyses de $\mathrm{N}_{2} \mathrm{O}$ sont effectuées à l'aide d'un chromatographe GIRDEL 30 équipé d'un détecteur à capture d'électrons (source radioactive $\mathrm{Ni}^{63}$ ) et d'une colonne remplie Porapak $\mathbf{Q}$. L'air ambiant sert d'étalon (310 ppbv).

\section{- Mesure de $\mathrm{H}_{2} \mathrm{~S}$}

L'hydrogène sulfuré est mesuré à l'aide d'un analyseur à fluorescence UV Environnement SA AF 41M, conçu pour la mesure des composés soufrés réduits totaux. L'appareil est constitué d'un convertisseur catalytique à haute température $\left(870^{\circ} \mathrm{C}\right)$ qui oxyde les composés réduits du soufre en $\mathrm{SO}_{2}$ (rack TRS). Le $\mathrm{SO}_{2}$ est ensuite détecté par fluorescence UV. La limite de détection de l'appareil est de $1 \mathrm{ppb}$, il est étalonné à l'aide d'un banc de calibration intégré muni d'un tube à perméation calibré d' $\mathrm{H}_{2} \mathrm{~S}$. L'appareil détecte donc, non sélectivement, l'ensemble des composés réduits du soufre: l'hydrogène sulfuré mais aussi les sulfures de méthyl et de carbone. L'hydrogène sulfuré est cependant largement dominant lors de la réduction des sulfates qui conduit à la formation de composés gazeux.

Les mesures de flux et de profils $d^{\prime} \mathrm{H}_{2} \mathrm{~S}$ dissous sont basées sur le même principe que les mesures de $\mathrm{CH}_{4}$ et $\mathrm{CO}_{2}$. Pour les flux, des échantillons d'air d'un volume de 1 litre, sont prélevés dans des sacs en téflon connectés à l'enceinte flottante, à l'aide d'un système d'aspiration sous vide non contaminant. Les sacs sont ensuite connectés en laboratoire à l'analyseur. La mesure est réalisée dans les 2 heures qui suivent le prélèvement. Pour la mesure $d^{\prime} \mathrm{H}_{2} \mathrm{~S}$ en solution, le dégazage de $100 \mathrm{ml}$ d'eau prélevée à l'aide d'une pompe péristaltique est réalisée dans une bouteille de 1 litre, préalablement vidée. Au moment de l'analyse, la bouteille est équilibrée à la pression atmosphérique et connectée à l'analyseur. L'intégration du signal obtenu permet de déterminer la quantité $d^{\prime} \mathrm{H}_{2} \mathrm{~S}$ dans la phase gazeuse. 
- Caractéristiques physico-chimiques de la colonne d'eau

Les caractéristiques physico-chimiques de la colonne d'eau sont mesurées directement, in situ, à l'aide de sondes spécifiques. Une sonde oxymétrique OM 96 WTW étalonnée avant chaque mesure à la saturation est utilisée pour la mesure de l'oxygène; le $\mathrm{pH}$ et la température sont mesurés à l'aide d'une sonde de terrain WTS IP 65 dont l'étalonnage est contrôlé avant chaque profil. Enfin sont également mesurés la conductivité (sonde type LF 92 WT) et le potentiel d'oxydoréduction au moyen d'une électrode spécifique couplée à un multimètre.

\section{3 ÉVOLUTION TEMPORELLE DES CONCENTRATIONS DE $\mathrm{CH}_{4}$, $\mathrm{CO}_{2}, \mathrm{D}^{\prime} \mathrm{H}_{2} \mathrm{~S}$ ET DE $\mathrm{N}_{2} \mathrm{O}$ DANS LA RETENUE}

L'évolution temporelle des caractéristiques physico-chimiques de la colonne d'eau, des profils de méthane, de gaz carbonique, d'hydrogène sulfuré et de protoxyde d'azote, dans la colonne d'eau a été suivie depuis la mise en eau sur les trois sites tests (CBN, FI et BPS) au cours des différentes campagnes. Sur le troisième site (BPS), nous disposons depuis mars 95 d'une mesure hebdomadaire des profils de $\mathrm{CH}_{4}$ et d' $\mathrm{O}_{2}$ dans la colonne d'eau.

\subsection{Méthane}

Les concentrations moyennes de $\mathrm{CH}_{4}$ dissous dans la colonne d'eau sont calculées par interpolation linéaire entre les points expérimentaux correspondant à des profondeurs de $0,0,5,1,2,5,10 \mathrm{~m}$ puis tous les $10 \mathrm{~m}$ jusqu'au fond du lac (fig. 1). Les résultats sont regroupés dans le tableau 1. Les profils de concentration de $\mathrm{CH}_{4}$ dans la retenue se caractérisent par une forte augmentation de mars à décembre 1994, qui se poursuit avec un taux moindre jusqu'en février-mai 1995 où des valeurs moyennes sur toute la hauteur de la colonne d'eau de l'ordre de $14 \mathrm{mg}$ $\left(\mathrm{CH}_{4}\right) . I^{-1}$ ont été atteintes à BPS. Les deux autres sites suivent exactement

Tableau 1. - Evolution des concentrations moyennes de méthane $\left(\mathrm{mg} . \mathrm{I}^{-1}\right.$ ) dans la colonne d'eau sur les trois sites suivis depuis la mise en eau.

Tableau 1. - Evolution of average methane concentrations (mg..$^{-1}$ ) in the water column at the three sites studied since reservoir filling.

\begin{tabular}{|c|c|c|c|c|c|c|c|c|}
\hline Conc. de $\mathrm{CH}_{4}$ & Mars 94 & Sep. 9 & I Déc. 94 & Fév. 95 & Mai 95 & Sep. 9 & Déc. 95 & Mars 96 \\
\hline Petit-Saut (BPS) & 0,37 & 6,57 & 12,18 & 13,88 & 13,90 & 8,60 & 13,05 & 7,90 \\
\hline Forêt inondée (FI) & 0,11 & 2,08 & 6,96 & - & 8,00 & 7,90 & - & - \\
\hline $\begin{array}{l}\text { Crique Bonne } \\
\text { Nouvelle (CBN) }\end{array}$ & 0,55 & 4,58 & 6,88 & - & 10,04 & 5,08 & 一 & 一 \\
\hline
\end{tabular}



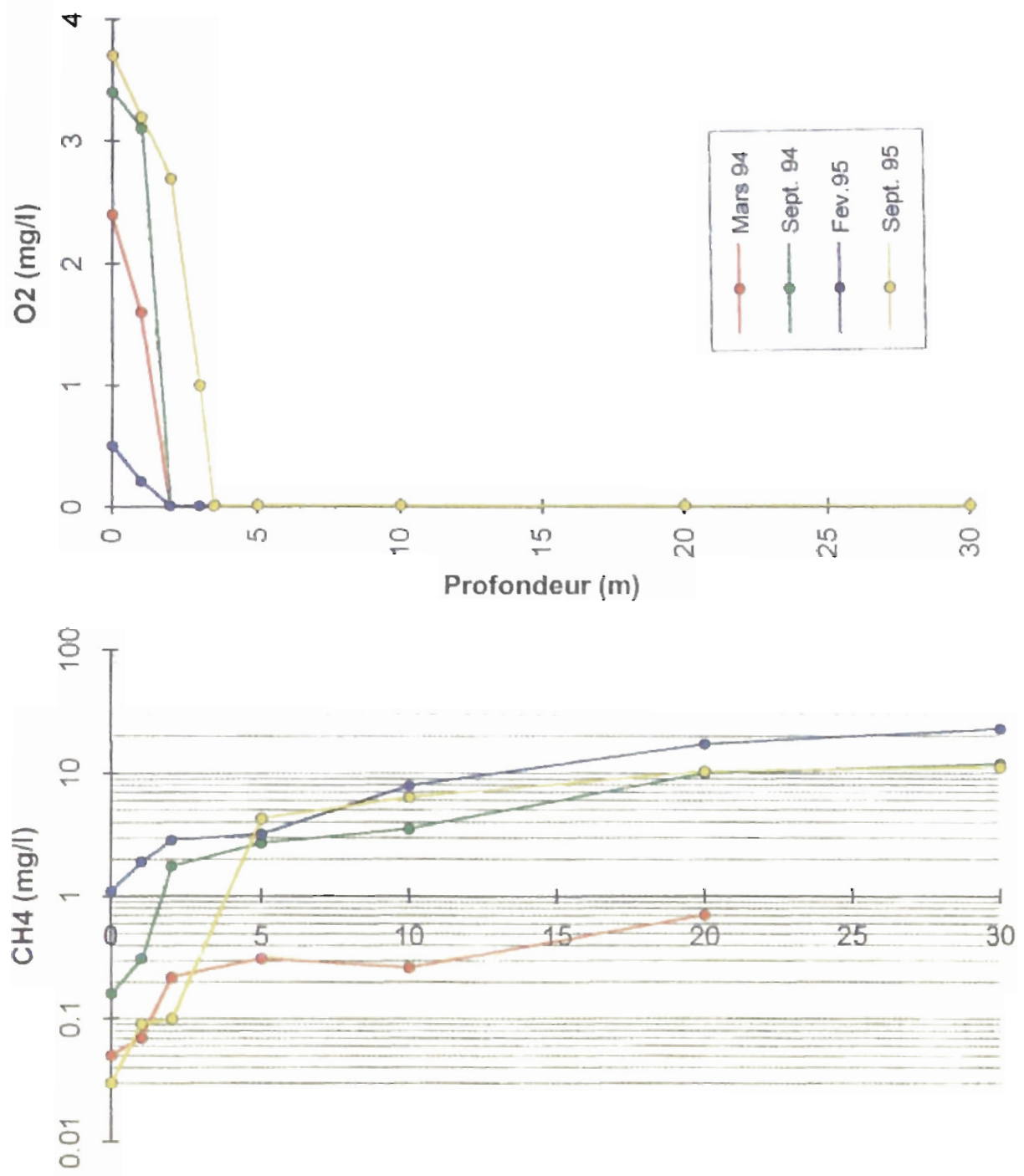

\section{Profondeur (m)}

Fig. 1. - Evolution des profils de méthane et d'oxygène dans la colonne d'eau à la station BPS de mars 1994 à septembre 1995.

Fig. 1. - Evolution of methane and oxygen profiles in the water column at BPS Station from March 1994 to September 1995. 
les mêmes évolutions temporelles avec des valeurs moyennes plus faibles car ils correspondent à des hauteurs de colonnes d'eau inférieures à celle de BPS (valeurs maximales observées de l'ordre de 8 à $10 \mathrm{mg}^{-1}{ }^{-1}$ ). Les concentrations diminuent ensuite après mai 1995 pour revenir à des valeurs moyennes de $8 \mathrm{mg}^{-l^{-1}}$ (BPS) en septembre 1995, du même ordre de grandeur que celles mesurées en septembre 94. A partir du mois de mars 1995 le suivi hebdomadaire du $\mathrm{CH}_{4}$ dissous à BPS fait apparaître des fluctuations de la concentration moyenne qui sont liées à la dynamique du lac et notamment aux varia- tions du débit entrant et sortant qui créent un effet de dilution des gaz dissous dans la masse d'eau (Galy-Lacaux et al., 1997b) (fig. 2). Le temps de rétention des eaux dans la retenue devient plus faible du fait de la saison des pluies. Le renouvellement rapide de la masse d'eau s'accompagne d'une réduction de l'accumulation des composés réduits, formés au fond de la retenue.

Les profils d'oxygène et de méthane mesurés à BPS, présentés sur la figure 1, montrent l'anticorrélation entre la concentration en méthane dissous et la teneur en oxygène dans l'eau. La présence d'oxygène dans la

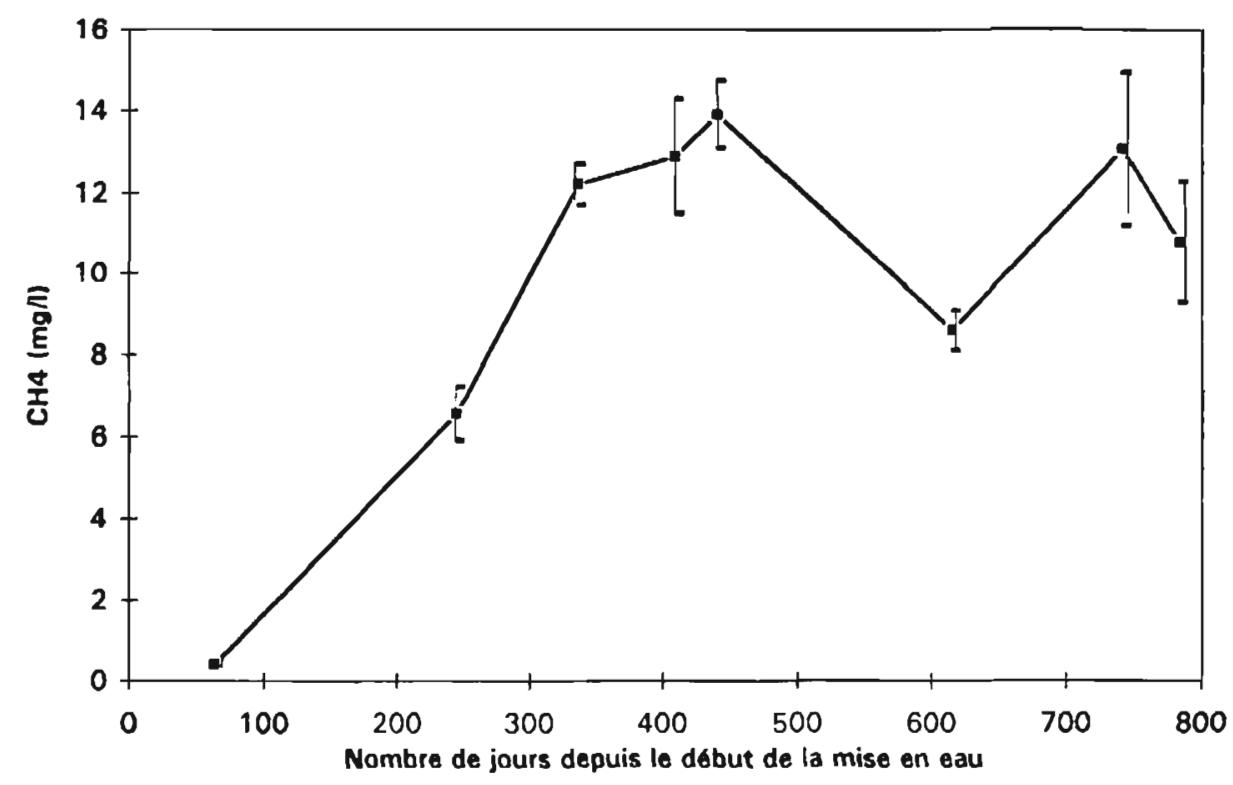

Fig. 2. - Variations de la concentration moyenne de méthane dissous dans la colonne d'eau à la station BPS depuis le début de la mise en eau. Les barres verticales représente l'écart type à la moyenne (le nombre de profils varie de $n=4$ à 10).

Fig. 2. - Variations of average concentration of dissolved methane in the water column at BPS stalion since reservoir filling. Vertical bars represent standard deviation (the number of vertical profiles is from 4 to 10). 
couche superficielle chaude de la retenue (épilimnion) est accompagnée d'une faible concentration en méthane dissous. L'hypolimnion, dont la profondeur varie, est anoxique et un fort gradient de méthane est observé à l'interface épi/hypolimnion. A partir de mars 1995, la concentration en oxygène a augmenté jusqu'à une valeur supérieure à $3 \mathrm{mg} \cdot \mathrm{I}^{-1}$, pendant que la concentration en méthane dans la couche de surface a diminué pour devenir inférieure à $0.1 \mathrm{mg} . \mathrm{l}^{-1}$. Au même moment, l'oxycline s'est déplacée vers une profondeur inférieure de l'ordre de $3 \mathrm{~m}$. La colonne d'eau des trois sites étudiés, est caractérisée par une absence totale d'oxygène dans l'hypolimnion et une couche oxique su- perficielle de l'ordre de $2 \mathrm{~m}$ d'épaisseur, dans laquelle la concentration en oxygène se trouve en dessous de la saturation avec au maximum 4 mg..$^{-1}$ (fig. 1).

L'évolution des concentrations de méthane et d'oxygene (fig. 3) dans la couche superficielle depuis le début de la mise en eau montrent la même corrélation inverse. La consommation quasi totale de l'oxygène dans la retenue est apparue quelques jours après la mise en eau du réservoir et s'est maintenue tout au long de la phase de remplissage avec une intensité variable. Un mélange partiel des eaux a réduit la stratification thermique en février 1995 (Richard, 1996), et cette période correspond à une

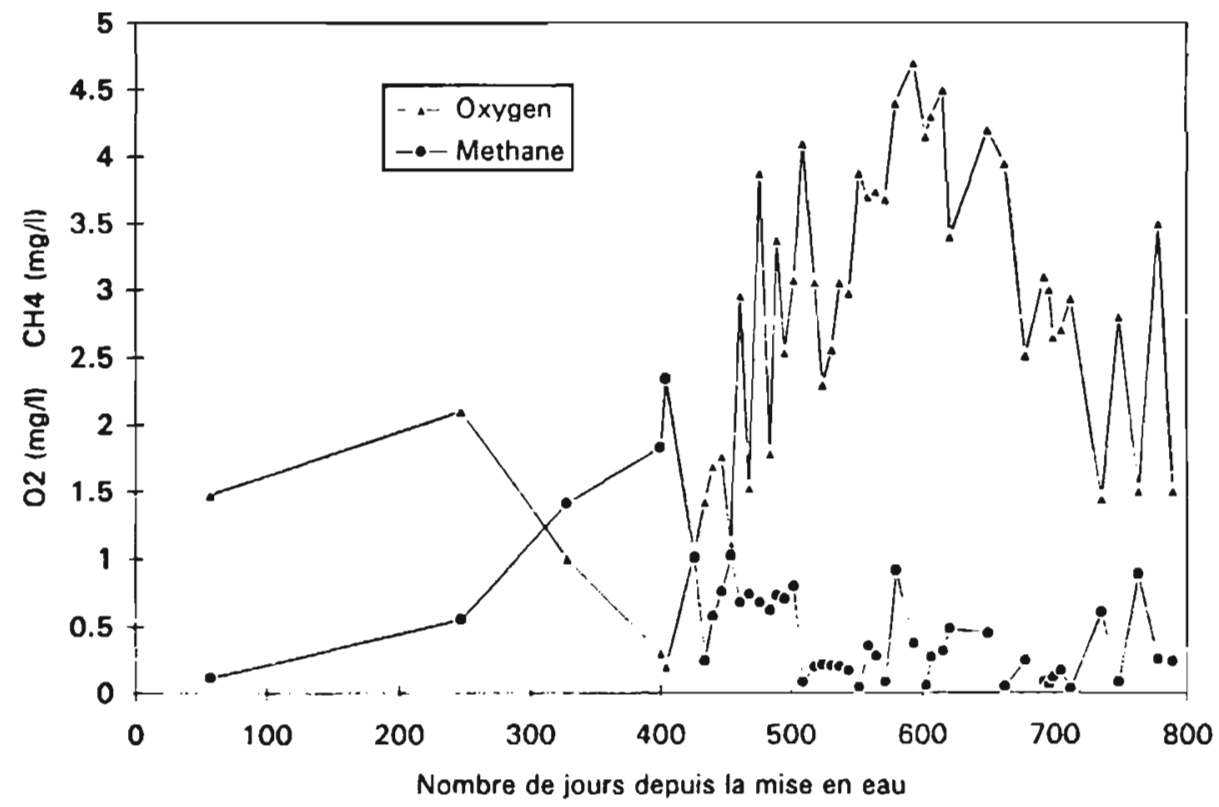

Fig. 3. - Evolution depuis la mise en eau de la concentration en méthane et en oxygene dans la couche de surface (0-2 m. station BPS).

Fig. 3. - Evolution of methane and oxygen concentrations within the surface layer $10-2 \mathrm{~m}$, BPS station). 
anoxie quasiment complète de la colonne d'eau avec des concentrations en méthane dissous dans la couche de surface $(0-2 \mathrm{~m})$ supérieures à

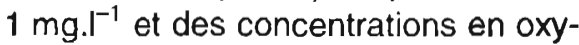
gène inférieures à $1 \mathrm{mg}^{-1}{ }^{-1}$.

Par ailleurs une bonne corrélation $(r=0,95)$ entre les concentrations moyennes de méthane dans la colonne d'eau et celles mesurées à $15 \mathrm{~m}$ est observée, montrant que cette dernière peut être considérée comme représentative de la colonne d'eau moyenne.

\subsection{Dioxyde de carbone}

Les profils de $\mathrm{CO}_{2}$ ont été mesurés sur les mêmes sites tests que le méthane et à partir des mêmes échantillons. L'évolution des concentrations en $\mathrm{CO}_{2}$ dissous a été suivie de façon moins régulière que le méthane en raison de son inertie chimique vis-àvis de la qualité de l'eau; cependant l'étude des concentrations en $\mathrm{CO}_{2}$ dissous est importante dans cette étude puisqu'elle permettra d'établir un bilan simplifié du carbone pour l'ensemble de la retenue.

L'évolution des concentrations en $\mathrm{CO}_{2}$ dissous n'ont pas suivi la même variation que celle du méthane. En surface de la retenue, les concentrations restent homogènes, de l'ordre de 15 à $20 \mathrm{mg} . \mathrm{I}^{-1}$ à $1 \mathrm{~m}$ de profondeur et ne subissent pas de brusque diminution. Au fond de la retenue, les concentrations ont augmenté pour atteindre des valeurs maximales en mai 1995 de l'ordre de $150 \mathrm{mg}^{-\mathrm{I}^{-1}}$. Les concentrations moyennes de $\mathrm{CO}_{2}$ dans la colonne d'eau montrent une évolution de $74 \mathrm{mg} . \mathrm{I}^{-1}$ en septembre 94 , avec un maximum en mai 95 de l'ordre de $100 \mathrm{mg.l}^{-1}$ avant de redescendre vers des valeurs de $80 \mathrm{mg} . \mathrm{I}^{-1}$ en septembre 95 , comparables à celles de septembre 94. Ces concentrations moyennes sont bien corrélées aux concentrations moyennes de méthane dans la colonne d'eau avec un rapport moyen constant de l'ordre de 9 , depuis le début du remplissage du réservoir (Le rapport pondéral moléculaire $\mathrm{C}-\mathrm{CO}_{2} / \mathrm{C}-\mathrm{CH}_{4}$ est égal à 3.3).

\subsection{Hydrogène sulfuré}

Les mesures des profils d' $\mathrm{H}_{2} \mathrm{~S}$ réalisées durant la campagne de décembre 1994 sur les 3 sites tests de la retenue montrent qu'il n'y a pas d' $\mathrm{H}_{2} \mathrm{~S}$ dans la couche de surface du lac. Les concentrations augmentent de façon significative avec la profondeur pour atteindre $50 \mu \mathrm{g} . \mathrm{I}^{-1}$ au fond du lac (fig. 4). Les concentrations massiques moyennes en $\mathrm{H}_{2} \mathrm{~S}$ dissous dans la colonne sont 1000 fois plus faibles que celles observées pour le méthane. Un exemple de profil obtenu à BPS est donné sur la figure 4 ; sur cette même figure sont reportés les profils d'ions sulfates et d'oxygène. Des valeurs similaires ont été mesurées il y a 20 ans par Brinkman et Santos (1974) dans des lacs naturels de la plaine Amazonienne et par Balon et Coche en 1974 dans le lac de barrage Kariba en Afrique Centrale. La réduction des sulfates se produit dans la plupart des 


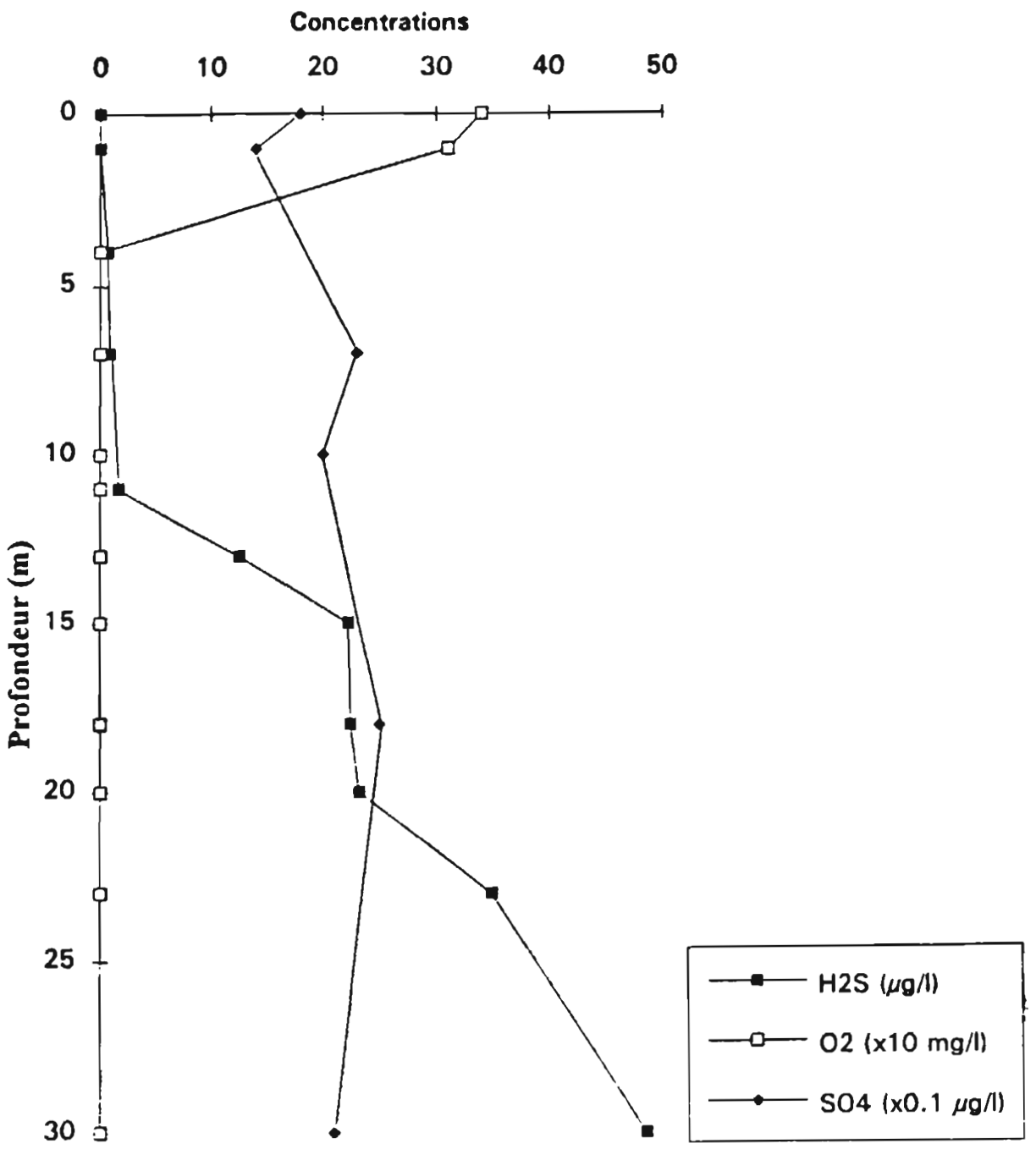

Fig. 4. - Exemple de profil d'hydrogène sulfuré mesuré en décembre 1994 à la station BPS associé au profil de sulfate et d'oxygene.

Fig. 4. - Example of hydrogen sulfide vertical profile measured in December 1994 at BPS station and associated with sulfate and oxygen profiles. 
lacs eutrophes et de façon générale avec une intensité moins importante que la méthanogenèse.

\subsection{Protoxyde d'azote}

Les profils de $\mathrm{N}_{2} \mathrm{O}$ mesurés principalement durant la campagne de mars 1994 indiquent qu'il n'y a pas ou très peu (au maximum $0.5 \mu \mathrm{g} . \mathrm{I}^{-1}$ sur toute la colonne d'eau) de $\mathrm{N}_{2} \mathrm{O}$ dissous dans la colonne d'eau. Les conditions anoxiques ne permettent pas la formation de nitrates; la concentration en nitrates mesurée dans la retenue est généralement nulle ne dépassant pas une dizaine de ppb dans la couche de surface où la nitrification est rendue possible par la présence d'oxgène. Ceci a été vérifié depuis le début de la mise en eau jusqu'en septembre 1995: aucune nitrification n'a été observée jusqu'à présent. D'autre part, les concentrations en ions ammonium qui étaient de l'ordre de 200 à $400 \mu \mathrm{g} . \mathrm{I}^{-1}$ en mars 1994 ont augmenté régulièrement pour atteindre des valeurs moyennes de l'ordre de $1000 \mu \mathrm{g} . \mathrm{I}^{-1}$ en septembre 95. Cette augmentation traduit la minéralisation importante qui se produit au fond de la retenue de façon continue et ces valeurs sont bien corrélées aux variations de conductivité au fond du lac (Richard, 1996). Les concentrations en ammonium dans l'eau à Petit-Saut sont proches de celles observées à Balbina au Surinam (Electronorte, 1993).
4 ÉMISSIONS ATMOSPHÉRIQUES À LA SURFACE DE LA RETENUE

\subsection{Mesures des flux diffusifs de méthane}

Les flux d'émission diffusifs de méthane déterminés à l'aide des enceintes flottantes ont été mesurés sur les mêmes sites et aux mêmes instants que les concentrations de gaz dissous dans la colonne d'eau. Les résultats sont présentés dans le tableau 2 pour l'ensemble des sites. Les flux de méthane à la surface de la retenue sont tous positifs et suivent la même évolution que les concentrations en méthane dissous dans l'eau de la retenue. Les flux les plus élevés sont mesurés à la station BPS et, sur la retenue les flux moyens ont suivi la même évolution que celle observée sur ce site. Les flux de méthane augmentent de façon quasi-exponentielle de 5 à $135 \mathrm{mg} \cdot \mathrm{m}^{-2} \cdot \mathrm{h}^{-1}$ entre mars 1994 et février 1995. En mars 1995, la décroissance de la concentration en méthane dissous dans la couche de surface est accompagnée par une décroissance des flux de méthane à la surface du lac. Les flux sont divisés par 100 entre février et mai 1995. La source de méthane ayant peu varié, si l'on se réfère aux concentrations moyennes dans la colonne d'eau ou aux concentrations au fond du lac; la diminution des flux de méthane pourrait être due à un développement important des bactéries méthanotrophes qui oxydent le méthane. 
Tableau 2. - Flux diffusif de méthane en $\mathrm{g}\left(\mathrm{CH}_{4}\right) \cdot \mathrm{m}^{-2} \cdot \mathrm{h}^{-1}$, à la surface du lac, sur les trois sites tests étudiés depuis le début de la mise en eau, durant les différentes campagnes de mesure. $\sigma$ représente l'écart type à la moyenne pour l'ensemble des données. Tableau 2. - Diffusive flux of methane in $\mathrm{g}\left(\mathrm{CH}_{4}\right) \cdot \mathrm{m}^{-2} \cdot \mathrm{h}^{-1}$, at the lake surface, at the three test sites studied since reservoir filling, during the different campaigns. $\sigma$ represents standard deviation for the whole data set.

\begin{tabular}{|c|c|c|c|c|c|c|}
\hline $\begin{array}{l}\mathrm{CH}_{4} \text { Flux } \\
\text { g. } \mathrm{m}^{-2} \cdot \mathrm{h}^{-1}\end{array}$ & , Mars 94 & Sept. 94 & Déc. 94 & Fév. 95 & Mai 95 & Sept. 95 \\
\hline Petit-Saut (BPS) & 0,005 & 0,020 & 0,108 & 0,135 & 0,002 & 0,003 \\
\hline $\begin{array}{l}\text { Forêt } \\
\text { inondée (FI) }\end{array}$ & 0,003 & 0,015 & 0,027 & - & $7,2010^{-4}$ & 0,005 \\
\hline $\begin{array}{l}\text { Crique Bonne } \\
\text { Nouvelle (CBN) }\end{array}$ & 0,007 & 0,013 & 0,010 & - & $2,210^{-4}$ & 0,004 \\
\hline Moyenne $(\sigma)$ & $\begin{array}{c}0,005 \\
(0,0015)\end{array}$ & $\begin{array}{c}0,013 \\
(0,005)\end{array}$ & $\begin{array}{c}0,048 \\
(0,013)\end{array}$ & $\begin{array}{l}0,135 \\
(0,02)\end{array}$ & $9,810^{-4}$ & $\begin{array}{c}0,004 \\
(0,002)\end{array}$ \\
\hline
\end{tabular}

Une expérience de mesure de consommation réelle de méthane dans la colonne d'eau a été effectuée en décembre 95 par le C.E.S.A.C (Centre d'Etudes des Systemes Aquatiques Continentaux) selon la procédure décrite et mise au point par Dumestre et Labroue en 1995. La consommation réelle de $\mathrm{CH}_{4}$ est maximale et presque totale au niveau de l'oxycline $(-4,5 \mathrm{~m})$; la vitesse d'oxydation observée est élevée $\left(0,425 \mathrm{mg}\left(\mathrm{CH}_{4}\right) / /\right.$.h $)$ ce qui dénote la présence d'une population méthanotrophique très dense ou très active. La raison pour laquelle ce développement s'intensifie à partir de mars 95 , soit plus d'un an après la mise en eau, n'est pas démontrée pour le moment. Cependant, une hypothèse sérieuse a été émise par le C.E.S.A.C. L'hydrogène sulfuré pourrait être un facteur d'inhibition pour le développement de ces bactéries et la diminution observée (liée à la dégradation rapide des protéines) en fin de remplissage aurait alors permis le développement de l'activité intense des populations méthanotrophiques (Dumestre et al., 1997). L'influence de l' $\mathrm{H}_{2} \mathrm{~S}$ sur des souches de méthanotrophes prélevées à Petit-Saut, sera testée en réalisant des croissances bactériennes en laboratoire.

\subsection{Emission spécifique sous forme de bulles de méthane}

Les flux d'émission sous forme de bulles ont été mesurés en mars et septembre 1994. Par la suite, il n'a plus été possible d'accéder aux zones peu profondes en forêt inondée pour des raisons de sécurité. Les résultats (tableau 3a) montrent un flux d'émission positif sur les sites de CriqueBonne-Nouvelle et en Forêt Inondée pour des profondeurs inférieures à $10 \mathrm{~m}$. Lors des deux campagnes de mesures, les flux de méthane sous 
Tableau 3a. - Flux de méthane lié au phénomène d'ébullition mesurés sur la retenue de Petit-Saut en mars et septembre 1994.

Tableau 3a. - Ebullitive methane fluxes measured on Petit-Saut reservoir in march and september 1994.

\begin{tabular}{|c|c|c|c|c|c|c|}
\hline \multirow{2}{*}{ Site } & \multicolumn{3}{|c|}{ Mars 1994} & \multicolumn{3}{|c|}{ Septembre 1994} \\
\hline & $n$ & $\begin{array}{l}\text { Profondeur } \\
\text { (m) }\end{array}$ & $\mid \begin{array}{c}\text { Flux de } \mathrm{CH}_{4} \\
\mathrm{mg} \cdot \mathrm{m}^{-2} \cdot \mathrm{j}^{-1}\end{array}$ & $\mathbf{n}$ & $\begin{array}{l}\text { Profondeur } \\
(\mathrm{m})\end{array}$ & \begin{tabular}{|} 
Flux de $\mathrm{CH}_{4}$ \\
$\mathrm{mg} \cdot \mathrm{m}^{-2} \cdot \mathrm{j}^{-1}$
\end{tabular} \\
\hline CBN (40 km du barrage) & 5 & $2-3$ & 1404 & 5 & $7-9$ & 240 \\
\hline FI 1 (20 km du barrage) & 5 & 6 & 936 & 5 & $5-6$ & 600 \\
\hline $\mathrm{FI} 2$ (5 km du barrage) & 5 & 15 & 0 & 5 & 25 & 0 \\
\hline BPS (300 m du barrage) & 5 & 19 & 0 & 5 & 31 & 0 \\
\hline DPS (50 $\mathrm{m}$ du barrage) & 5 & 10 & 0 & 5 & 10 & 0 \\
\hline
\end{tabular}

forme de bulles sont nuls à l'approche du barrage, à la station BPS et contre la digue.

Le phénomène d'effervescence n'apparait que lorsque les profondeurs sont limitées. II est probable que lorsque les profondeurs sont plus importantes, les bulles ne se forment pas ou subissent une redissolution à l'interface liquide-gaz pendant leur remontée vers la surface du lac. De plus, cette remontée n'est possible que si les conditions de pression dans la colonne d'eau le permettent. II faut rappeler que l'émission de méthane sous forme de bulles est fonction de nombreux paramètres : la profondeur, la température, la quantité de substrat organique, le vent ou toute perturbation favorisant le départ des bulles des sédiments vers la surface d'eau (Keller et al., 1994). Des travaux ont montré que dans certains cas (pour un lac donné), le phénomène de diffusion prédomine sur le phénomène d'effervescence ou inversement. II est difficile d'établir une telle relation dans le cas du lac de Petit-Saut, compte tenu du faible nombre de mesures, qui se limitent au début de la mise en eau. Nous pouvons cependant comparer les mesures effectuées à Petit-Saut avec celles réalisées sur le lac Gatun au Panama (tableau 3b). Les valeurs diminuent lorsque la profondeur augmente et deviennent nulles pour des profondeurs supérieures à $10 \mathrm{~m}$. Les flux de méthane liés à l'effervescence sont du même ordre de grandeur sur ces deux lacs tropicaux et sont très importants comparés aux flux diffusifs mesurés à la surface du lac, après mars 1995.

\subsection{Mesures des flux d'émission de gaz carbonique}

Les flux de $\mathrm{CO}_{2}$ mesurés sur les mêmes emplacements et aux mêmes instants que ceux de méthane sont présentés dans le tableau 4. On constate que contrairement aux flux 
Tableau 3b. - Comparaison des flux de méthane liés au phénomène d'ébullition sur le Lac Gatun (Panama) et sur la retenue de Petit-Saut.

Tableau 3b. - Comparison of bubbling methane fluxes from Gatun Lake (Panama) and from Petit-Saut reservoir.

\begin{tabular}{|c|c|c|c|}
\hline Site & Profondeur (m) & $\begin{array}{c}\text { Flux moyen } \\
\text { d'ébullition }\left(\mathrm{mg} \mathrm{m}^{-2} \mathrm{j}^{-1}\right) \mathrm{i}\end{array}$ & $\left(\mathrm{mg} \mathrm{\textrm {m } ^ { - 2 }} \Gamma^{-1}\right)$ \\
\hline \multicolumn{4}{|l|}{ Lac Gatun (Panama) } \\
\hline Peu profond & $0,5-2,6$ & 967 & 450 \\
\hline Moyennement profond & $3-5,5$ & 394 & 82 \\
\hline Profond & $6,9-9,8$ & 70 & 20 \\
\hline \multicolumn{4}{|l|}{ Petit-Saut (Guyane) } \\
\hline Peu profond & $2-3$ & 1400 & 650 \\
\hline Moyennement profond & $5-6$ & 770 & 300 \\
\hline Moyennement profond & $7-9$ & 240 & 90 \\
\hline Profond & $>10$ & 0 & \\
\hline
\end{tabular}

Tableau 4. - Evolution des flux moyens de $\mathrm{CO}_{2}$ sur les trois sites étudiés depuis la mise en eau. $\sigma$ représente l'écart type à la moyenne pour l'ensemble des données.

Tableau 4. - Evolution of average $\mathrm{CO}_{2}$ fluxes on the three sites studied since resenoir filling. $\sigma$ represents standard deviation for the whole data set.

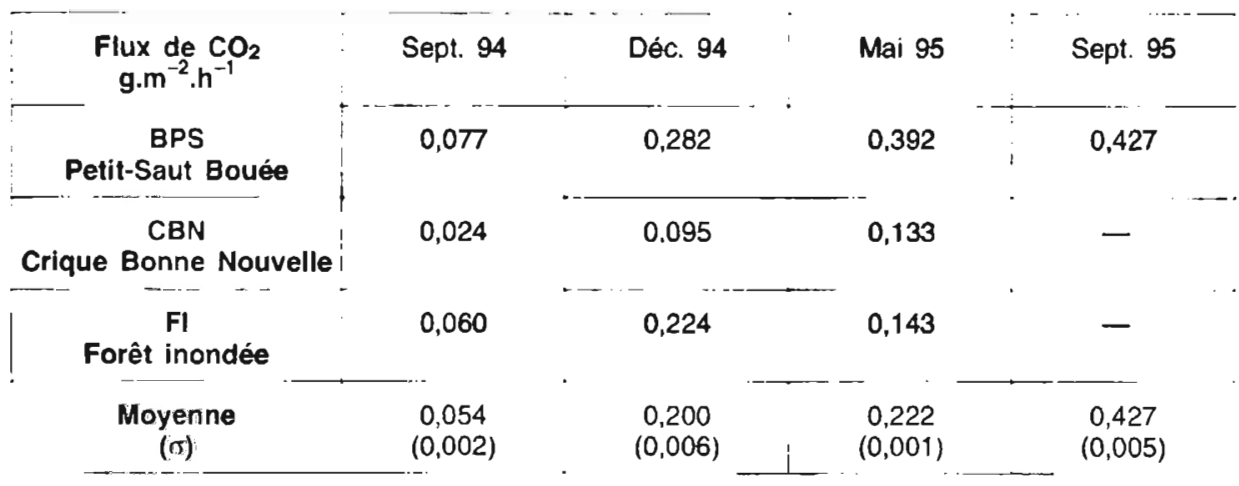

de $\mathrm{CH}_{4}$, les flux de $\mathrm{CO}_{2}$ ne présentent pas de diminution spectaculaire. Les flux moyens à la surface de l'eau, augmentent régulièrement à partir de $54 \mathrm{mg}\left(\mathrm{CO}_{2}\right) \cdot \mathrm{m}^{-2} \cdot \mathrm{h}^{-1}$ en septembre 1994 jusqu'à environ $400 \mathrm{mg}\left(\mathrm{CO}_{2}\right) \cdot \mathrm{m}^{-2} \cdot \mathrm{h}^{-1}$ en septembre 1995. A la station BPS le rapport de flux $\mathrm{F}\left[\mathrm{CO}_{2}\right] \mathrm{F}\left[\mathrm{CH}_{4}\right]$ passe de 2,6 en décembre 94 à 106 en septembre 95 alors que le rapport des concentrations moyennes dans la colonne reste, dans le même temps, voisin de 9 . Ceci confirme le rôle essentiel joué par l'oxydation biotique du méthane dans la réduction des flux diffusifs de méthane à l'interface eau-atmosphere. 


\subsection{Mesures des flux d'émission d'hydrogène sulfuré et de protoxyde d'azote}

Les mesures des flux d' $\mathrm{H}_{2} \mathrm{~S}$ ont été principalement réalisées lors de la campagne de mesure de décembre 1994 à la surface de l'eau sur les sites précédemment mentionnés. Les flux mesurés sont nuls indiquant qu'il n'y a pas d'émission d'hydrogène sulfuré à la surface de l'eau. Ce résultat est en accord avec l'absence totale d' $\mathrm{H}_{2} \mathrm{~S}$ dans la couche de surface de la retenue. La forte solubilité d' $\mathrm{H}_{2} \mathrm{~S}$ et les teneurs somme toute faibles, ne dépassant $0,05 \mathrm{mg} . \mathrm{l}^{-1}$ au fond de la retenue $d^{\prime} \mathrm{H}_{2} \mathrm{~S}$ en solution peuvent expliquer ce résultat.

Les flux de $\mathrm{N}_{2} \mathrm{O}$ mesurés principalement lors de la campagne de mars 1994 sont très faibles. Les valeurs obtenues sont comprises entre 0 et $13,7 \mu \mathrm{g}(\mathrm{N}) \cdot \mathrm{m}^{-2} \cdot \mathrm{h}^{-1}$. Par ailleurs, nous avons vérifié que les émissions de NO étaient nulles à la surface de la retenue.

\section{5 ÉMISSIONS GAZEUSES EN AVAL DE LA DIGUE}

Les gaz dissous contenus dans l'eau turbinée sont en majeure partie libérés dans l'atmosphère par dégazage au pied de la digue. Le barrage a été doté d'un seuil à lame déversante qui provoque, en sortie des turbines une chute d'eau de $4 \mathrm{~m}$ dont le but est de faciliter les échanges gazeux eau-air. Un contrôle assidu de l'efficacité de cette installation est effectué par le Laboratoire de PetitSaut. Les mesures montrent que, en moyenne, plus de $80 \%$ du méthane dissous est immédiatement libéré après la chute d'eau, laquelle permet également la saturation en oxygène. Au cours des campagnes de mai et septembre 1995, des expériences spécifiques ont été effectuées, d'une part au niveau du seuil pour déterminer les concentrations dans l'air ambiant, d'autre part sur le Sinnamary en aval pour étudier les différentes composantes des flux de méthane ainsi que les phénomènes de consommation d'oxygène dans la rivière par l'oxydation du méthane résiduel.

\subsection{Concentrations de $\mathrm{CH}_{4}, \mathrm{CO}_{2}$ et $\mathrm{H}_{2} \mathrm{~S}$ dans l'air ambiant sur le site du barrage}

En mai 95, des mesures de concentration de $\mathrm{CH}_{4}, \mathrm{CO}_{2}$ et $\mathrm{H}_{2} \mathrm{~S}$ dans l'air ambiant ont été effectuées à proximité du seuil à lame déversante, dans le panache des gaz libérés. L'émission par dégazage des rejets est très localisée; des concentrations très élevées ont été mesurées juste au dessus du seuil. Une dizaine de mesures effectuées le 16/5/95 au dessus du seuil sont présentées dans le tableau 5. Des concentrations de 1 à 4,5 ppmv d'hydrogène sulfuré dans l'air sont observées suivant le prélèvement. Simultanément, l'analyse de méthane et de dioxyde de carbone atmosphérique a été réalisée. Les 
Tableau 5. - Concentrations en ppm de méthane, de dioxyde de carbone et d'hydrogène sulfuré mesurées en mai 95 dans le panache des gaz émis au pied du seuil de réoxygénation.

Tableau 5. - Concentrations (in ppm) of methane, carbon dioxide and hydrogen sulfide measured in march 1995 at the bottom of the reoxygenation waterfall.

\begin{tabular}{|c|c|c|c|c|}
\hline Echantillons & {$\left[\mathrm{CH}_{4}\right] \mathrm{ppmv}$} & {$\left[\mathrm{CO}_{2}\right] \mathrm{ppmv}$} & {$\left[\mathrm{CH}_{4}\right]\left[\mathrm{CO}_{2}\right]$} & {$\left[\mathrm{H}_{2} \mathrm{~S}\right] \mathrm{ppmv}$} \\
\hline 1 & 1631 & & & 2,37 \\
\hline 2 & 1078 & 1305 & 0,82 & 1,42 \\
\hline 3 & 3360 & 3041 & 1,1 & 1,68 \\
\hline 4 & 1177 & 1367 & 0,86 & 4,55 \\
\hline 5 & 3362 & 3061 & 1,09 & 1,78 \\
\hline 6 & 2507 & 2392 & 1,04 & 3,73 \\
\hline 7 & 1387 & 1521 & 0,91 & 2,24 \\
\hline 8 & 800 & 1102 & 0,73 & 1,55 \\
\hline 9 & 648 & 1085 & 0,597 & 0,27 \\
\hline \begin{tabular}{c|c|c|c|c|} 
Moyenne (ppmv) \\
(écart type)
\end{tabular} & 1772 & 1859 & 0,95 & 2,17 \\
\hline (988) & $(786)$ & $\ldots$ & $(1,2)$ \\
\hline
\end{tabular}

concentrations qui varient de 650 à 3400 ppm de méthane et de 1100 à $3100 \mathrm{ppm}$ de dioxyde de carbone dans l'air prouvent l'efficacité du seuil à lame déversante. Le rapport des concentrations $\left(\mathrm{CO}_{2} / \mathrm{CH}_{4}\right)$ dans l'air du panache de dégazage est voisin de 1 alors que dans l'eau il est en moyenne de 9. Ceci confirme une efficacité du dégazage plus grande pour le méthane que pour le gaz carbonique liée à la différence de solubilité des deux gaz. Les mesures dont nous disposons dans l'eau de la rivière en aval du seuil montrent que seulement $50 \%$ du $\mathrm{CO}_{2}$ présent dans l'eau turbinée est libéré instantanément dans l'atmosphère.

\subsection{Méthane et oxygène dissous dans le Sinnamary. Consommation d'oxygène}

Trois expériences sur la rivière ont été effectuées au cours des campagnes de mai et septembre 1995, dans le but d'étudier le comportement des gaz en solution dans la rivière. Les concentrations de $\mathrm{CH}_{4}$ et $\mathrm{O}_{2}$ dissous, et les flux de méthane à l'interface eau-air ont été mesurés simultanément en un nombre de points suffisants entre le barrage et Pointe Combi, pour avoir une description détaillée de l'évolution des phénomènes. Afin de réaliser une évaluation précise des termes 
d'échanges, les valeurs expérimentales ont été interpolées de manière à déterminer l'évolution du flux de méthane en surface avec un pas de $1 \mathrm{~km}$; les résultats obtenus le 1/09/95 sont présentés sur la figure 5 . Une diminution rapide de l'oxygène et une augmentation du méthane en surface dans les deux premiers kilomètres après le barrage est observée. Le flux de méthane augmente également, proportionnellement à la concentration dans l'eau de surface. Ceci peut être due à un mélange imparfait de l'eau en sortie du seuil, la couche de surface étant mieux oxygénée et dégazée, ou à une hétérogénéité horizontale due à l'effet du clapet de surface qui amène des eaux de bonne qualité en rive gauche. Le mélange intervient par turbulence au cours du transport. A partir du troisième $\mathrm{km}$, les concentrations en oxygène et méthane dissous chutent; les flux de $\mathrm{CH}_{4}$ diminuent également. Les concentrations et flux de méthane s'annulent généralement entre 20 et $30 \mathrm{~km}$ après le barrage. La concentration en $\mathrm{O}_{2}$ dissous se stabilise alors autour de $3 \mathrm{mg} \cdot \mathrm{l}^{-1}$. L'intégration donne la perte cumulée de méthane par diffusion. La connaissance de la concentration moyenne de méthane dans la colonne d'eau, en amont de la retenue, et du débit turbiné, permet de réaliser le bilan complet de ce gaz après passage de l'eau dans les turbines. Ce bilan est reporté dans le tableau 6, pour les expériences de mai et septembre 95.

Selon les débits et les concentrations initiales dans le lac, 80 à $90 \%$ du méthane dissous est libéré dans l'atmosphère au niveau du seuil. Sur la fraction restante, seulement $20 \%$ est émis par diffusion à l'interface eau-air, le reste disparaît par oxydation biologique dans la rivière. Cette oxydation intervient dans les 20 à 30 premiers $\mathrm{km}$ et est probablement la cause principale de la diminution de l'oxygène dissous observée.

A partir de l'ensemble des mesures sur le Sinnamary aval, il est possible d'établir une relation entre consommation d'oxygène et oxydation du méthane. Nous avons calculé ces deux termes à partir des mesures réalisées au cours du suivi hebdomadaire à Kerenroch et Pointe Combi de mars à septembre 1995 par le laboratoire Environnement de Petit-Saut. La relation entre $\mathrm{D}\left[\mathrm{O}_{2}\right]$ (consommation d'oxygène) et $\mathrm{D}\left[\mathrm{CH}_{4}\right]$ (perte de méthane) est représentée sur la figure 6. Le rapport molaire $\mathrm{D}\left[\mathrm{O}_{2}\right] / \mathrm{D}\left[\mathrm{CH}_{4}\right]$ est de 1,7 , ce qui est proche de l'équilibre stoechiométrique de la réaction (biologique) d'oxydation: $\mathrm{CH}_{4}+2 \mathrm{O}_{2}$ $\rightarrow \mathrm{CO}_{2}+2 \mathrm{H}_{2} \mathrm{O}$ selon laquelle l'oxydation d'une mole de méthane consomme 2 moles d'oxygène. II faut noter toutefois que la perte de méthane par diffusion gazeuse (qui représente environ $20 \%$ de la perte totale) et les apports par les affluents sur le cours aval du Sinnamary ne sont pas pris en compte dans ce calcul. II en est de même de l'alimentation en oxygène par diffusion à l'interface. La régression linéaire entre consommation d'oxygène et perte de méthane $\left(\mathrm{D}\left[\mathrm{O}_{2}\right]=1,26^{*}\right.$ $\left.\mathrm{D}\left[\mathrm{CH}_{4}\right]+1,60\right)$ montre une corrélation 


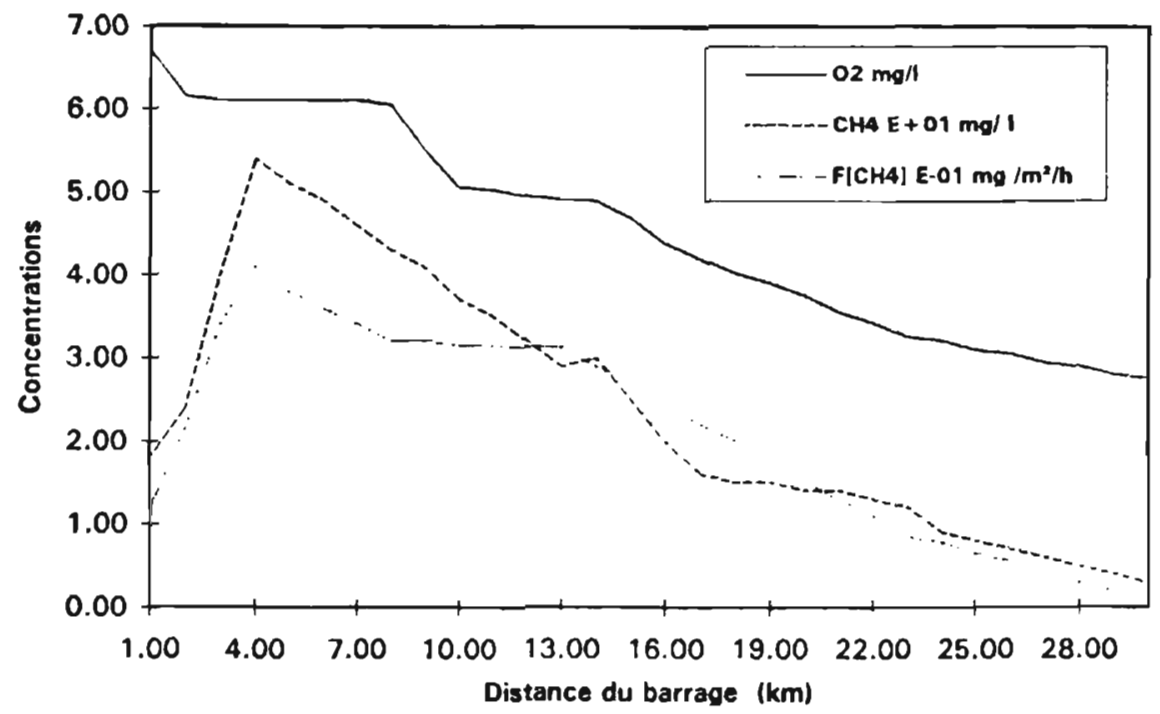

Fig. 5. - Variations de la concentration en oxygène et en méthane et des flux de méthane en surface dans le Sinnamary aval le 1/09/95 (valeurs interpolées pour le calcul du flux).

Fig. 5. - Variations of oxygen and methane concentrations and of methane fluxes at the surface in the Sinnamary river dowstream of the dam on September 1, 1995 (values interpolated for flux calculation).

Tableau 6. - Devenir du méthane dans le Sinnamary aval (Expériences de mai et septembre 1995).

Tableau 6. - Fate of methane in the Sinnamary river downstream of the dam (Experiments of may and september 1995).

\begin{tabular}{|c|c|c|c|}
\hline Expériences en aval de la digue & 18 mai 95 & $1^{\text {er }}$ sept. 95 & 11 sept. 95 \\
\hline$\left[\mathrm{CH}_{4}\right]$ amont $(\mathrm{mg} / \mathrm{l})$ & 13,3 & 4,09 & 6,93 \\
\hline$\left[\mathrm{CH}_{4}\right]$ aval $(\mathrm{mg} / \mathrm{l})$ & 1,10 & 0,66 & 0,56 \\
\hline Débit moyen Journalier $\left(\mathrm{m}^{3} / \mathrm{s}\right)$ & 100 & 220 & 225 \\
\hline Flux moyen $\mathrm{CH}_{4}$ aval $(\mathrm{mg} / \mathrm{m} / \mathrm{h})$ & $20,0(0-20 \mathrm{~km})$ & $20,8(0-30 \mathrm{~km})$ & $14,0(0-30 \mathrm{~km})$ \\
\hline a $-\mathrm{CH}_{4}$ exporté de la retenue ( $\left.\mathrm{T} / \mathrm{jour}\right)$ & 114,9 & 77,89 & 134,88 \\
\hline $\begin{array}{l}\mathrm{CH}_{4} \text { dégazé à la chute (T/jour) } \\
(\% \text { de a) }\end{array}$ & $\begin{array}{l}105,4 \\
(92 \%)\end{array}$ & $\begin{array}{l}65,26 \\
(84 \%)\end{array}$ & $\begin{array}{l}123,86 \\
(92 \%)\end{array}$ \\
\hline $\begin{array}{l}\mathrm{b}-\mathrm{CH}_{4} \text { entraîné dans la rivière (T/jour) } \\
(\% \text { de a) }\end{array}$ & $\begin{array}{l}9,5 \\
(8 \%)\end{array}$ & $\begin{array}{c}12,63 \\
(16 \%)\end{array}$ & $\begin{array}{l}11,02 \\
(8 \%)\end{array}$ \\
\hline $\begin{array}{l}\mathrm{CH}_{4} \text { émis dans l'atmosphère (T/Jour) } \\
(\% \text { de b) }\end{array}$ & $\begin{array}{c}1,9 \\
(20 \%)\end{array}$ & $\begin{array}{c}3,0 \\
(24 \%)\end{array}$ & $\begin{array}{c}2,0 \\
(18 \%)\end{array}$ \\
\hline $\begin{array}{l}\mathrm{CH}_{4} \text { oxydé dans la rivière (T/jour) } \\
(\% \text { de b) }\end{array}$ & $\begin{array}{c}7,63 \\
(80 \%)\end{array}$ & $\begin{array}{c}9,63 \\
(76 \%)\end{array}$ & $\begin{array}{c}9,02 \\
(82 \%)\end{array}$ \\
\hline
\end{tabular}

Le flux d'émission de méthane est calculé à partir du flux moyen en considérant une largeur moyenne de la rivière de $200 \mathrm{~m}$ et la distance entre la digue ef le point ou le flux s'annule. La quantité de méthane oxydé est calculée par différence entre la quantité non dégazée (entraînée dans la rivière) et le flux émis dans l'atmosphere. 


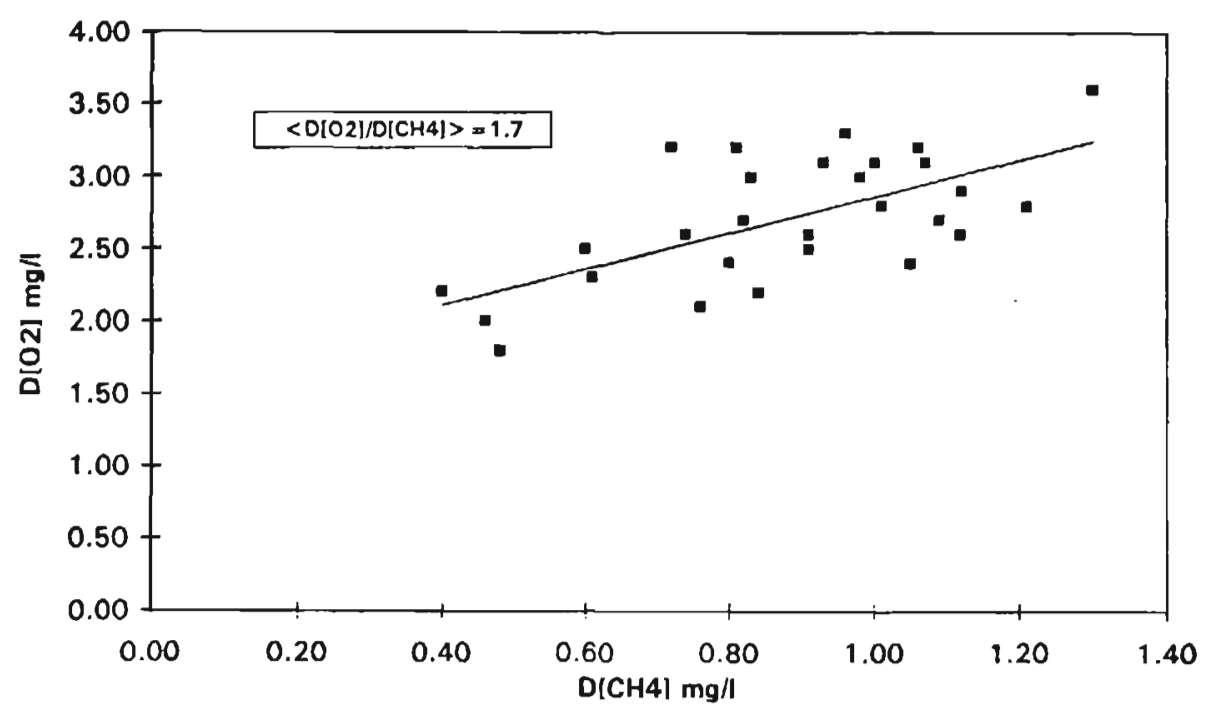

Fig. 6. - Corrélation entre les variations des concentrations de méthane et d'oxygène dans la riviere Sinnamary (mars à septembre 1995).

Fig. 6. - Correlation between methane and oxygen concentrations in the Sinnamary river (from march to september 1995).

significative $(r=0,65)$ mais la pente de la droite de régression n'est que de 1,26 avec une ordonnée à l'origine de $1,6 \mathrm{mg}\left(\mathrm{O}_{2}\right) \cdot \mathrm{I}^{-1}$. Ces résultats ne permettent pas de conclure sur une consommation d'oxygène dans la rivière exclusivement liée à l'oxydation du méthane résiduel. II faut noter cependant que lorsque les teneurs en méthane dissous sont nulles la consommation d'oxygène cesse.

\section{BILAN DU CARBONE DE LA RETENUE DE PETIT-SAUT, 2 ANS APRÈS LA MISE EN EAU}

L'évolution des phénomènes physico-chimiques, liée à la dégradation de la matière organique dans la retenue de Petit-Saut, a été étudiée et quantifiée sur une période de 2 ans. L'in- tégration de l'ensemble des données expérimentales de flux d'émission gazeuse de $\mathrm{CH}_{4}$ et de $\mathrm{CO}_{2}$ et de gaz dissous dans la masse d'eau $\left(\mathrm{CH}_{4}\right.$, $\mathrm{CO}_{2}$ ) a permis d'établir un bilan simplifié du carbone de la retenue.

La formation de méthane et de gaz carbonique consécutive à la dégradation de la matière organique dans la retenue, conduit après diffusion dans la colonne d'eau, à une émission dans l'atmosphère au niveau de la surface du lac; ainsi qu'à une émission par dégazage des rejets en aval du barrage. Le bilan entre production et perte est fermé en permanence par le terme d'accumulation des gaz dissous dans la masse d'eau. Depuis la mise en eau du barrage, le bilan de carbone au niveau de la retenue, si on néglige la production primaire et 
les apports externes de carbone, se résume à:

\section{Production $(\mathrm{CH} 4+\mathrm{CO} 2)=$ Flux en surface (ébullition + diffusion) + Flux exporté en aval + Stock (1)}

où la production (limitée au $\mathrm{CH}_{4}$ et au $\mathrm{CO}_{2}$ ) représente la perte de carbone du stock initial, lequel inclue la biomasse épigée et le carbone du sol. Pour réaliser ce bilan nous pouvons déterminer les trois termes de droite de l'équation (1) à partir des mesures effectuées entre mars 1994 et janvier 1996.

\section{Estimation des flux liés à la diffu- sion}

L'estimation des émissions moyennes à la surface du lac suppose la connaissance du flux moyen journalier et de la surface émettrice. L'évolution journalière de la surface peut être calculée à partir du bilan hydrologique. Si $V_{i}$ est le volume d'eau stocké au jour $\mathrm{i}$, et $\mathrm{z}_{\mathrm{i}}$ le niveau moyen de la retenue, la surface Si est calculée suivant l'équation (2).

$$
S_{i}=\frac{V_{i}-V_{i-1}}{z_{i}-z_{i-1}}
$$

La variation temporelle de surface de la retenue ainsi calculée est représentée sur la figure 7. Les flux d'émis-

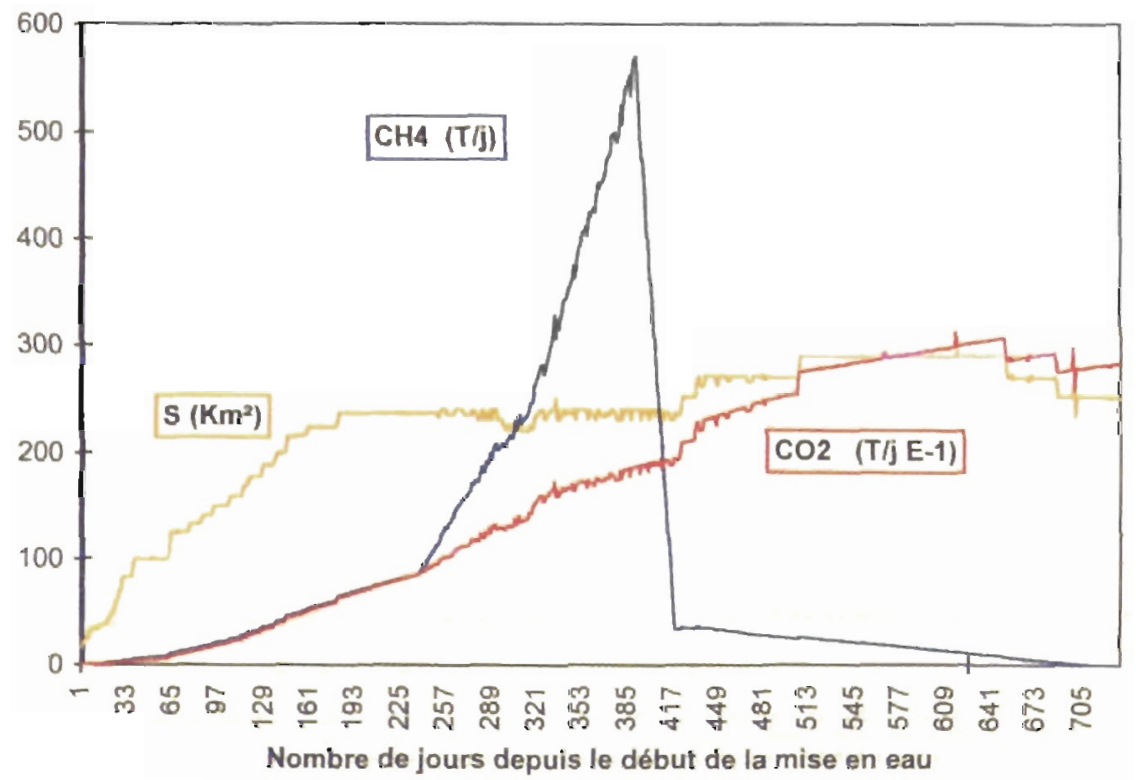

Fig. 7. - Variations joumalières de la superficie de la retenue, des flux de méthane et des flux de dioxyde de carbone à la surface du lac, depuis la mise en eau. Emissions cumulées: 65000 t $\left(\mathrm{CH}_{4}\right), 1200000 \mathrm{t}\left(\mathrm{CO}_{2}\right)$.

Fig. 7. - Daily variations of reservoir area, methane and carbon dioxide fluxes since reservoir filling Cumulated emissions : $65000 \mathrm{t}\left(\mathrm{CH}_{4}\right), 1200000 \mathrm{t}\left(\mathrm{CO}_{2}\right)$. 
sion moyens sont calculés à partir des valeurs expérimentales obtenues sur les différents sites (tableau 2 et 4 ) et moyennées, après interpolation linéaire pour obtenir des données journalières $\Phi_{1}$. Le flux journalier total est alors $\mathrm{Fi}=\Phi_{i}^{*} \mathrm{~S}_{\mathrm{i}}$. L'évolution temporelle du flux diffusif de méthane est représentée sur la figure 7 . Le flux cumulé depuis la mise en eau s'écrit:

$$
F\left[\mathrm{CH}_{4}\right]=\sum_{i=1}^{i=n} \Phi i * S i
$$

L'émission de $\mathrm{CH}_{4}$ est caractérisée par une augmentation quasi-exponentielle, entre janvier 1994 et févriermars 1995, atteignant une valeur maximale de $570 \mathrm{t}\left(\mathrm{CH}_{4}\right) \cdot \mathrm{j}^{-1}$. Elle subit ensuite une diminution due à l'atténuation du flux en surface probablement due à l'oxydation biologique par les bactéries méthanotrophes au niveau de l'oxycline. Cette variation n'apparaît pas dans l'émission de $\mathrm{CO}_{2}$ qui a cru régulièrement depuis la mise en eau avant de se stabiliser au bout de 18 mois autour d'une valeur moyenne de l'ordre de $2800 \mathrm{t.j}^{-1}$. Le flux cumulé de janvier 1994 à janvier 1996 de la retenue de Petit-Saut est estimée à $65 \pm 3010^{3}$ tonnes de $\mathrm{CH}_{4}$ et à $1200 \pm 50010^{3}$ tonnes de $\mathrm{CO}_{2}$.

\section{Estimation des flux liés au phénomène d'ébullition}

Malgré, le faible nombre de mesures de flux liées au phénomène d'ébullition, réalisées sur la retenue de Petit-Saut, nous pouvons estimer ce flux, sachant que les valeurs mesurées sont cohérentes avec l'étude réalisée par Keller et al. en 1994 sur le lac Gatun au Panama (tableau 3b). Le flux total est égal à la somme des flux obtenus par classe de profondeur d'eau, en multipliant le taux d'émission mesuré, par la surface correspondante déterminée à partir des bilans hydrologiques. Cette estimation décrite dans le tableau 7 montre que l'émission de méthane sous forme de

Tableau 7. - Estimation du flux moyen de méthane par phénomène d'ébullition sur la retenue de Petit-Saut.

Tableau 7. - Assessment of average bubbling flux of methane from the Petit-Saut reservoir.

\begin{tabular}{|c|c|c|c|}
\hline $\begin{array}{l}\text { Profondeur d'eau } \\
(\mathrm{m})\end{array}$ & $\begin{array}{c}\text { Surface } \\
\text { correspondante } \\
\left(\mathrm{km}^{2}\right)\end{array}$ & $\begin{array}{l}\text { Flux moyen } \\
\text { d'ébullition } \\
\left(\mathrm{mg} \mathrm{m}^{-2} \mathrm{i}^{-1}\right)\end{array}$ & $\begin{array}{c}\text { Emission de } \mathrm{CH}_{4} \\
\text { par bullage } \\
\left(\mathrm{t} . \mathrm{j}^{-1}\right)\end{array}$ \\
\hline $0-3$ & 58 & $1400 \pm 650$ & $81 \pm 38$ \\
\hline $4-6$ & 40 & $770 \pm 300$ & $31 \pm 12$ \\
\hline $7-9$ & 32 & $240 \pm 90$ & $8 \pm 3$ \\
\hline & & Total & $120 \pm 53$ \\
\hline
\end{tabular}


bulles est comparable à l'émission de méthane liée à la diffusion en surface du lac. Elle représenterait $120 \pm 53 \mathrm{t.j}^{-1}$ soit un flux cumulé de $87 \pm 3810^{3}$ tonnes de méthane émis sous formes de bulles pendant deux ans sur la retenue de Petit-Saut. Les bulles de biogaz contenant essentiellement du méthane $(80 \%)$, ce type d'émission a peu d'importance dans le flux de $\mathrm{CO}_{2}$ (60. $\left.10^{3} \mathrm{t}\right)$.

\section{Estimation des quantités de $\mathrm{CH} 4$ et $\mathrm{CO}_{2}$ exportées en aval}

Le calcul de la perte de carbone sous forme de $\mathrm{CH}_{4}$ et de $\mathrm{CO}_{2}$, consécutive aux rejets d'eau en aval du barrage par la vidange de fond, les turbines ou l'évacuateur de crues, suppose que l'on connaisse l'évolution temporelle des concentrations dans l'eau du lac au niveau de la prise d'eau, et le débit journalier cumulé. Les mesures de gaz dissous et de flux en aval, ont montré que la plus grande partie du méthane $(80 \%)$ et la moitié du $\mathrm{CO}_{2}$ sont libérées immédiatement au niveau du seuil (section 5). L'essentiel du méthane non dégazé, entraîné dans la rivière est oxydé en $\mathrm{CO}_{2}$ par les bactéries méthanotrophes, seul environ $20 \%$ du méthane résiduel est libéré dans l'atmosphère; le devenir du $\mathrm{CO}_{2}$ entraîné dans la rivière n'a pas été étudié. Le calcul de la perte en aval de la retenue se résume alors à la sommation, depuis la mise en eau, des quantités quotidiennement exportées, qui sont égales au produit de la concentration moyenne dans la colonne d'eau en amont de la digue $\left(\mathrm{g} \cdot \mathrm{m}^{-3}\right)$ par le débit relâché chaque jour $\left(\mathrm{m}^{3} \cdot \mathrm{j}^{-1}\right)$.

La quantité de méthane rejetée varie de 40 à plus de 500 tonnes par jour en fonction des variations du débit et des concentrations (figure 8). Le rejet cumulé deux ans après la mise en eau du barrage atteint environ $88 \pm 3010^{3}$ tonnes de $\mathrm{CH}_{4}$. La quantité de $\mathrm{CO}_{2}$ exportée de la retenue par les rejets d'eau en aval depuis la mise en eau du barrage est estimé à partir du rapport constant des concentrations moyennes $\left(\mathrm{CO}_{2} / \mathrm{CH}_{4}\right)$ dans l'eau et est de l'ordre de $800 \pm 13010^{3}$ tonnes de $\mathrm{CO}_{2}$.

Pour déterminer les quantités de méthane et de dioxyde de carbone introduites dans le réservoir, des échantillons d'eau ont été prélevées dans des rivières en amont de la retenue. Une concentration moyenne de $\mathrm{CO}_{2}$ égale à $10 \pm 2 \mathrm{mg}^{-1} \mathrm{l}^{-1}$ a été mesurée tandis que la concentration en $\mathrm{CH}_{4}$ dissous est inférieure à la limite de détection de la méthode de mesure. La quantité de $\mathrm{CO}_{2}$ dissous introduite dans le réservoir est estimée à partir de cette valeur et du débit entrant et est égale à $140 \pm 4010^{3}$ tonnes.

\section{Evolution du stock de $\mathrm{CH}_{4}$ et $\mathrm{CO}_{2}$ dissous dans la retenue}

Le calcul du stock de $\mathrm{CH}_{4}$ et $\mathrm{CO}_{2}$ s'appuie sur une intégration étendue à toute la masse d'eau, des concentrations des gaz en solution. Ces concentrations varient en fonction de la profondeur, présentant pour les deux gaz des gradients négatifs depuis le fond vers la surface (du fait de leur production au niveau du sé- 
diment). La quantité totale de gaz en solution dépend donc à la fois de ces concentrations $\mathrm{C}(\mathrm{z})$ et de la hauteur de la colonne d'eau qui varie continûment sur toute la surface du lac. La quantité totale de gaz stocké dans le réservoir est calculée à l'aide de l'expression suivante:

$$
\begin{aligned}
& Q=\sum_{i=1}^{t} \Delta S_{i} \cdot \frac{1}{10} \cdot \int_{0}^{z_{\ell_{i} i}} C(z) \cdot d z(\Delta S \text { en } \\
& \left.\mathrm{cm}^{2}, Q \text { en } \mathrm{mg}\right)
\end{aligned}
$$

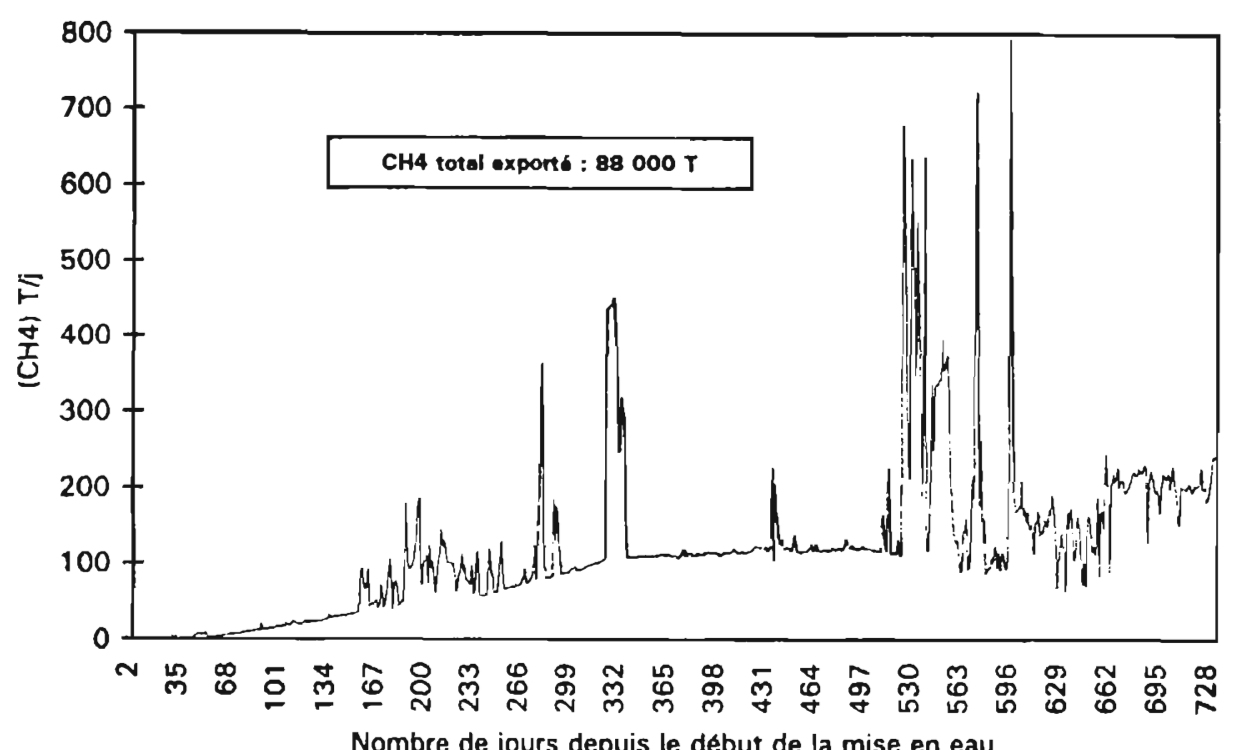

Fig. 8. - Variations journalières des émissions de méthane par dégazage des rejets en aval du barrage depuis le début de la mise en eau (Station: seuil à lame déversante).

Fig. 8. - Daily variations of methane emissions from released water degassing downstream of the dam, since reservoir filling (Station: waterfall).

Tableau 8. - Evolution des stocks de gaz dissous dans la retenue de Petit-Saut entre février 94 et décembre 95.

Tableau 8. - Evolution of dissolved gas pools in the Petit-Saut reservoir between february

\begin{tabular}{|c|c|c|c|c|c|c|c|}
\hline $\begin{array}{l}\text { Gaz Unité : } \\
10^{3} \text { Tonnes }\end{array}$ & Fév. 94 & Sept. 94 & Déc. 94 & Fév. 95 & Mai 95 & Sept. 95 & Déc. 95 \\
\hline $\mathrm{CH}_{4}$ & 1,07 & 12,2 & 20,9 & 20,9 & 30,14 & 23,14 & 25 \\
\hline $\mathrm{CO}_{2}$ & & 120,1 & 107,8 & & 204,13 & 137,6 & 210 \\
\hline C- $-\mathrm{CH}_{4}$ & 0,8 & 9,1 & 15,7 & 15,7 & 22,6 & 17,5 & 18,7 \\
\hline $\mathrm{C}-\mathrm{CO}_{2}$ & & 32,7 & 29,3 & & 55,7 & 37,5 & 57 \\
\hline$\Sigma \mathrm{C} \mathrm{CH}+\mathrm{CO}_{2}$ & & 41,8 & 44,9 & & 78,3 & 55 & 76 \\
\hline
\end{tabular}
1994 and december 1995. 
où $\Delta \mathrm{Si}$ correspond à la superficie de la retenue recouverte par une hauteur d'eau égale à $z_{0}=z_{f}-z_{i}, z_{f}$ étant le niveau de la retenue (profondeur maximum) pour laquelle le calcul est effectué et $C(z)$ représente les profils moyens de concentration de $\mathrm{CH}_{4}$ ou de $\mathrm{CO}_{2}$ calculés sur la base de l'ensemble des données mesurées au cours d'une campagne.

Les quantités totales de gaz en solution dans la masse d'eau et leur évolution de février 1994 à décembre 1995 sont donnés dans le tableau 8. D'après ces résultats, le stock de carbone sous forme de $\mathrm{CH}_{4}$ et de $\mathrm{CO}_{2}$ dissous dans la retenue aurait atteint une valeur maximale en mai 95 (ou entre février et mai). En septembre 95, la décroissance du stock est amorcée, puis des fluctuations probablement dues aux variations des débits turbinés sont observées. La valeur du stock en décembre 1995 de l'ordre de 76000 tonnes de carbone sera retenue pour le calcul du bilan deux ans après la mise en eau.

\section{Bilan du carbone}

La somme des quantités de carbone exportées de la retenue par émissions gazeuses à la surface du lac, par les rejets d'eau en aval, et du stock résiduel dans la retenue, permet d'établir le bilan du carbone deux ans après la mise en eau du réservoir et de déterminer la perte du stock initial (tableau 9). Comme nous l'avons souligné ce bilan reste partiel, certains termes n'étant pas pris en compte; on peut néanmoins supposer qu'ils sont mineurs par rapport aux termes évalués.

L'émission à la surface du lac reste le terme le plus important du bilan en dépit de la diminution depuis mars 1995 de l'émission de méthane. Ceci est dû aux flux de $\mathrm{CO}_{2}$ qui restent importants et qui sont sans doute renforcés par la transformation $\mathrm{CH}_{4} \rightarrow \mathrm{CO}_{2}$ dans l'eau de surface. La perte de méthane est néanmoins dominée par le dégazage des rejets immédiatement à l'aval du barrage. Seule une faible fraction (moins de $20 \%$ ) du méthane rejeté est oxydée en $\mathrm{CO}_{2}$ dans la rivière. Le stock de gaz en solution a tendance à diminuer après avoir atteint un maximum en février-mai 95 et représente 76000 tonnes de carbone sous forme de $\mathrm{CH}_{4}$ et $\mathrm{CO}_{2}$ dissous.

La biomasse épigée du réservoir est estimée à environ $170 \mathrm{t}(\mathrm{C}) \cdot \mathrm{ha}^{-1}$. Une partie significative de cette biomasse n'est pas submergée, cependant la fraction facilement biodégradable (feuilles et petites branches) tombe et se retrouve présente dans l'eau du réservoir peu de temps après la mise en eau. Elle se décompose donc en anaérobiose. La fraction restante, principalement sous forme de composés ligno-cellulosiques, sera dégradée très difficilement sur plusieurs dizaines d'années. Le stock de carbone soumis à la décomposition anaérobie doit tenir compte du carbone des sols. Le carbone contenu dans le sol a été estimé à $100 \mathrm{t}(\mathrm{C}) \cdot \mathrm{ha}^{-1}$ à l'aide de la base de données CDIC 
Tableau 9. - Bilan du carbone de la retenue de Petit-Saut 2 ans après la mise en eau. Tableau 9. - Carbon budget of Petit-Saut reservoir 2 years after reservoir filling.

\begin{tabular}{|c|c|c|c|}
\hline $\begin{array}{c}\text { Emission cumulée } \\
\text { ou stock en } \\
\text { janvier } 96 \\
\left.\text { ( } \times 10^{3} \text { tonnes }\right)\end{array}$ & $\mathrm{CH}_{4}$ & $\mathrm{CO}_{2}$ & $\begin{array}{l}\text { Carbone libéré } \\
\qquad \mathrm{C}= \\
\mathrm{C}-\mathrm{CH}_{4}+\mathrm{C}-\mathrm{CO}_{2}\end{array}$ \\
\hline $\begin{array}{l}\text { Emission diffusive à } \\
\text { la surface du lac }\end{array}$ & $65 \pm 30$ & $1200 \pm 500$ & $380 \pm 140$ \\
\hline $\begin{array}{l}\text { Emission par ébulli- } \\
\text { tion à la surface }\end{array}$ & $87 \pm 38$ & $60 \pm 30$ & $81 \pm 36$ \\
\hline $\begin{array}{l}\text { Perte par Rejets en } \\
\text { aval }\end{array}$ & $88 \pm 30$ & $800 \pm 130$ & $280 \pm 60$ \\
\hline $\begin{array}{l}\text { Stock de gaz dissous } \\
\text { dans la retenue }\end{array}$ & $25 \pm 5$ & $210 \pm 60$ & $76 \pm 20$ \\
\hline $\begin{array}{l}\text { Gaz dissous introduit } \\
\text { dans le réservoir }\end{array}$ & 0 & $-140 \pm 30$ & $-30 \pm 10$ \\
\hline $\begin{array}{c}\text { Total = perte de } \\
\text { carbone }\end{array}$ & $270 \pm 100$ & $2100 \pm 800$ & $800 \pm 300$ \\
\hline
\end{tabular}

Les incertitudes sur les différents termes du bilan de carbone sont estimées à partir des incertitudes sur l'échantillonage et les analyses chimiques (10\%), les incertitudes sur le bilan hydrologique et les débits $(10 \%)$. La principale source d'erreur provient de l'extrapolation des mesures sur les trois stations tests à l'ensemble de la retenue. L'incertitude relative des mesures de flux est estimée à partir de l'écart type.

Numeric Data Collection (Martin, Marietta Energy Systems Inc. 1986); une partie significative de ce carbone est facilement dégradable comme les invertébrés ou la biomasse microbienne du sol. La quantité totale de matière organique soumise à la décomposition est donc de l'ordre de $270 \mathrm{t}(\mathrm{C}) \cdot \mathrm{ha}^{-1}$ dans la retenue de Petit-Saut.

Le carbone total perdu, 2 ans après la mise en eau, est évalué à environ $800 \pm 30010^{3}$ tonnes de carbone pour une surface inondée qui atteint désormais $300 \mathrm{~km}^{2}$. La perte de carbone par la retenue serait donc de l'ordre de $27 \mathrm{t}(\mathrm{C}) \cdot \mathrm{ha}^{-1}$, ce qui repré- sente $10 \%$ du stock total de carbone et probablement une fraction significative du stock initial de carbone organique facilement biodégradable.

\section{CONCLUSION}

Les résultats obtenus lors des sept campagnes de mesures effectuées sur la retenue hydroélectrique de Petit-Saut, en Guyane, ont permis de quantifier et d'étudier les mécanismes d'émission de la production de biogaz $\left(\mathrm{CO}_{2}\right.$ et $\left.\mathrm{CH}_{4}\right)$ liée à la décomposition de la biomasse submergée. La consé- 
quence principale, sur le plan aquatique, de la production de méthane, résultant de la décomposition anaérobie de la matière organique au niveau du sédiment, est la consommation d'oxygène liée à l'oxydation biologique du méthane dans le réservoir et dans la rivière en aval du barrage. Ce processus affecte considérablement la qualité de l'eau et pour palier à cette désoxygénation un seuil à lame déversante a été construit en aval immédiat des turbines dans le but de favoriser le dégazage du méthane dissous dans l'eau. Deux années après le début de la mise en eau, une fraction significative (27 tonnes par hectare) du carbone stocké dans la biomasse facilement dégradable a été dégradée. L'émission de méthane à la surface du lac par diffusion à l'interface air-atmosphère était dominante la première année de remplissage et fût considérablement réduite par l'oxydation biologique du méthane dans la couche d'eau de surface. Les émissions gazeuses sont maintenant dominées par le dégazage de l'eau en sortie des turbines et par l'émission sous forme de bulles.

L'émission de méthane et de dioxyde de carbone par une retenue d'eau artificielle pourrait contribuer de façon significative à l'effet de serre additionnel. Les résultats obtenus peuvent aider à évaluer la source de gaz à effet de serre représentée par les retenues hydroélectriques. Compte tenu du nombre de barrages existant (plus de 37000 grands barrages en 1986 (CIBG, 1988)) et du développement prévisible de l'hydroé- lectricité dans les régions tropicales (comme au Brésil par exemple (Fearnside, 1995)), cette source pourrait être significative dans le bilan atmosphérique du méthane.

\section{REFERENCES BIBLIOGRAPHIQUES}

Balon, A. and Coche A.G. 1974. Lake Kariba : a Man-Made Tropical Ecosystem in Central Africa, edited by Dr Junk W., Publishers the Hague, 164-182.

Bartlett K. B., Crill P.M., Sebacher D.I., Hariss R.C., Wilson J.O. and Mellack J.M. 1988. Methane flux from the central Amazonian floodplain, J. Geophys. Res, 93, 1571-1582.

Brinkman W.L.F. and de M. Santos U. 1974. The emission of biogenic hydrogen sulfide from Amazonian floodplain lakes, Tellus, 26, 261-267.

CDIC Numeric Data Collection, 1986. Worldwide organic soil carbon and nitrogen data, NDP-O18, Oak Ridge National Laboratory, $133 \mathrm{p}$.

Chamberland A. 1992. Production de gaz à effet de serre résultant de l'aménagement de grands réservoirs au nord ouest du Québec, $9^{e}$ congrès mondial de l'air pur, Montréal.

Cicerone R.J. and Oremland R.S. 1988. Biogeochemical aspects of atmospheric Methane, Global Biogeochemical Cycles, 2 (4), 299-327.

$\mathrm{CIGB}, 1988$. Commission Internationale des Grands Barrages, Registre mondial des grands barrages (édition de 1983, mise à jour en 1988).

Conrad R. 1989. Control of methane production in terrestrial ecosytems, in Exchanges of Trace Gases Between Terrestrial Ecosystems and the Atmosphere, M.O. Andreae an D.S. Schimel Editors, J Wyley and Sons.

Delmas R.A., Servant J., Tathy J.P., Cros B. and Labat M. 1992. Sources and sinks of methane and carbon dioxide exchanges in mountain forest in equa- 
torial Africa, Journal of Geophysical research, 97(6), 6196-6179.

Devol A.H., Richey J.E., Clark W.A., King S.L. and Martinelli L.A. 1988. Methane emissions to the troposphere from the amazon floodplain, J. Geophys. Res., 93, 1583-1592.

Duchemin E., Lucotte M., Camuel R. et Chamberland A. 1995. Production of the greenhouse gases $\mathrm{CH}_{4}$ and $\mathrm{CO}_{2}$ by hydroelectric reservoirs of the boreal region. Global Biogeochemical Cycles, vol. 9, n 4, p. 529-540.

Dumestre J.F. et Labroue L. 1995. Aménagement hydroélectrique de PetitSaut (Guyane) : bilan des respirations aérobies et anaérobies dans un lac de barrage équatorial. Conséquences sur la désoxygénation de l'eau, l'émission de gaz traces et de composés toxiques, Rapport scientifique $n^{\circ} 1$ convention GP 7573 EDF-CNEH, 36 p.

Dumestre J.F, Labroue L., Galy-Lacaux C., Reynaouard C. et Richard S. 1997. Biomasse et activité bacterienne dans la retenue et à l'aval du barrage de Petit-Saut (Guyane) : influence sur les émissions de méthane et la consommation d'oxygène. Hydroécol. Appl. 9 (1/2) : pp. 139-167.

Eletronorte, 1993. Diagnostico de qualidade da agua. Fases rio, enchimento e resevatorio. Rapport Eletronorte, Brasilia, DF, $28 p$.

Fearnside P.M. 1995. Hydroelectric dams as sources of greenhouse gases, Environ. Conserv., 22 (1), 7-19.

Galy-Lacaux C., Delmas R., Jambert C., Dumestre J.F., Labroue L., Cerdan P., Richard S. 1996, Emission de méthane et consommation d'oxygene dans la retenue de Petit-Saut en Guyane, Comptes rendus à l'académie des sciences: Tome 322, Série IIA, no 12, p. 1013-1019.

Galy-Lacaux C. 1996. Modification des échanges de constituants mineurs atmosphériques liées à la création d'une retenue hydroélectrique. Impact des barrages sur le bilan du méthane dans l'atmosphère. Thèse de Doctorat de l'Université Paul-Sabatier (Toulouse III), décembre 1996.

Galy-Lacaux C., Delmas R., Jambert C., Dumestre J.F., Labroue L. 1997a Gaseous emissions and oxygen consumption in hydroelectric dams. A case study in French Guyana. Global Biogeochemical Cycles, sous presse.

Galy-Lacaux C.R. Delmas, Richard S. and Ceradan P. 1997b. Etude de l'évolution à moyen terme du méthane dissous dans le lac et en aval du Barrage de Petit-Saut. Etude des mesures du suivi 1996, Rapport Scientifique $\mathrm{n}^{\circ} 5$, EDFCNEH (avril 1997).

Heide J. 1982. Filling phase limnology of a man-made in the humid tropics. lake Brokopondo, offsetdrukkerij Kanter B.V. (ed), Albasserdam. p. 1-428.

Keller M. and Stallard R.F. 1989. Two methods for measuring methane emission from a tropical lake, Limnology and Oceanography.

Keller M. and Stallard R.F. 1994. Methane Emission by bubbling from Gatun Lake Panama. J. Geophys. Res., 99, 83078319.

Kelly C.A., Rudd J.M.W, St Louis V.L. and Moore T. 1994. Turning attention to reservoir surfaces, a neglected area in greenhouse study, Eos Trans. AGU, 75, 332-333.

Khalil M.A.K. 1995. Greenhouse gases in the earth atmosphere, in Encyclopedia of Environmental Biology, vol. 2, Academic Press Inc., 251-265.

Oremland S. and Culbertson C.W. 1992. Importance of methane-oxidizing bacteria in the methane budget as revealed by the use of a specific inhibitor, letters to Nature, vol. 356.

Pereira A. 1994. Contribution à l'étude de la qualité des eaux des retenues amazoniennes : application à la retenue de Tucurui (Brésil), Doctorat de l'Ecole Nationale des Ponts et Chaussées.

Richard S. 1996. La mise en eau de la retenue de Petit-Saut (Guyane Française): Hydrochimie du fleuve Sinna- 
mary avant la mise en eau, de la retenue pendant la mise en eau et du fleuve en aval, Thèse de Doctorat, Université de Marseille I.

Rudd J.W.M. et Taylor C.D. 1980. Methane cycling in aquatic environments. Adv. Aquat. Microbiol., 2 : 77-150.

Rudd J.W.M., Harris R., Kelly C.A. and Hecky R.E. 1993. Are Hydroelectric reservoirs significant sources of greenhouse gases, Ambio, 22(4), 246-248.

Shine K. P., Derwent R.G., Wuebbles D.J. and Morcette J.J. 1990. Radiative forcing on climate, in Climate Change, The IPCC Scientific Assessment, 45-68.

Sissakian C. 1992. EDF/CNEH. Présentation de la retenue de Petit-Saut en Guyane Française: cartographie partition de la retenue - volumes et surfaces - intégration paysagère, Hydroécologie Appliquée, 1, 121-132.

Sigg. L.W., Stumm and Behra P. 1992. Chimie des milieux aquatiques, Chimie des eaux naturelles et des interfaces dans l'environnement, Ed. Masson.

Smith L.K. and Lewis W.M. 1992. Methane emissions from Orinico River Floodplain, Venezuela, ASLO 92, Aquatic core Sci. Mtg., Santa Fe, NM, Feb. 9-14.

Tathy J.P., Delmas R.A., Cros B., Marenco A. and Servant J. 1992. Methane emissions from flooded forest in Central Africa, J. Geophys. Res., 97 (D6), 6159-6168.

Wassmann R.U.G. Thein, Withicar M.J., Rennenberg $H$., Seiler W. and Junk W.J. 1992. Methane emissions from the amazonian floodplain : characterization and transport. Global Biogeochemical Cycles, 6(1), 3-13. 